\title{
Inhaltliche und methodische Einführung
}

\author{
Hintergrund: Film und Kunst seit den 199oer Jahren
}

In seiner Kritik zum dritten Skulptur Projekt in Münster im Jahr 1997, das parallel zur documenta X in Kassel stattfand, lobt Gottfried Knapp in der Süddeutschen Zeitung die Arbeit von Douglas Gordon als gelungene Wiederbelebung einer Fußgängerunterführung am Hindenburgplatz (heute Schlossplatz) in Münster. Zwanzig Jahre nachdem Joseph Beuys auf die städtebauliche Ruine durch eine Skulptur aufmerksam gemacht hatte, für die er einen großen Hohlraum unterhalb einer Rampe mit Talg nachbaute und damit den NichtOrt des Übergangs in organisches Material übertrug, verweist auch Gordons Arbeit auf die Funktion des Gangs als Passage. In einer Videoinstallation wird auf eine freistehende Leinwand von beiden Seiten je ein Film projiziert, Der Exorzist von William Friedkin und Das Lied von Bernadette von Henry King, einerseits die Geschichte einer Teufelsaustreibung und andererseits die Beschreibung der religiösen Erleuchtung der Heiligen Bernadette. Knapp interpretiert die Arbeit treffend als Überschneidung der transgressiven Kraft des Kinos mit dem tatsächlichen Durchschreiten eines realen Ortes, der durch die filmische Parallelisierung eine symbolische Bedeutung erhält:

Die Leinwand wird im Untergrund also zu einem Tor in den Himmel wie in die Hölle; sie wird zur leuchtenden Sperre in einem Verlies, aus dem der Passant nur tastend ins Freie findet. Selten sind die physischen und psychischen Dimensionen des Kinos und die apokalyptischen Verirrungen des modernen Städtebaus so mit Händen zu greifen gewesen wie in dieser Kunst-Installation. ${ }^{1}$

In anschaulicher Weise kommen hier zwei zentrale Aspekte der Kunst der 199oer Jahre zusammen: die Anknüpfung an die site specifity der 1970er Jahre und der Trend der Videokunst zu aufwändigen Installationen, die sich mit den Rezeptionsweisen des Films und seinen narrativen und psychologischen Komponenten auseinandersetzen. Wie Holger Kube Ventura in seiner 2001 erschienenen Studie zur Kunst der 199oer Jahre zusammenfassend beschreibt, löst in dieser Zeit eine Repolitisierung und neue »soziologische Form« die

1 Gottfried Knapp, »Zehn Jahre sind keine Epoche - Die Skulptur-Projekte in Münster stehlen der documenta mit Phantasie und Witz die Schau«, in: Süddeutsche Zeitung Nr. 143, 25.6.1997, S. 13-14, S. 13 . 
objektzentrierte Kunst der 198oer Jahre ab. ${ }^{2}$ Boomten in den 198oer Jahren die klassischen Gattungen Malerei und Skulptur, die beispielsweise mit der Malerei der Neuen Wilden gut »ausstell- und verkaufbare Exponate $\aleph^{3}$ produzierten, sowie ein »künstlerischer Tenor, der unter Begriffen wie Affirmation, Subjektivismus und Ironie subsummierbar sei ${ }^{4}$, so sind die Arbeiten der folgenden Dekade von einer Ausweitung und Ausdifferenzierung der Medien gekennzeichnet. Nicht mehr die Bindung an ein bestimmtes Medium, sondern gerade die Unterordnung der künstlerischen Mittel unter den »Text« und die Entwicklung aus dem »theoretischen, institutionellen oder politischen Kontext $\aleph^{5}$ heraus kennzeichnen die aufkommende Installationskunst. Die 199oer Jahre gelten als das Jahrzehnt, in dem Film- und Videoprojektionen den Ausstellungsraum übernehmen. Nicht nur die Arbeit mit Video, die seit den späten 196oer Jahren die Konzept- und Performancekunst prägte - man denkt an Bruce Nauman, Nam June Paik oder auch VALIE EXPORT -, sondern auch computergenerierte Bilder und Simulationen, Einflüsse des Musikvideos und der Popkultur bestimmen die Medienkunst (zum Beispiel bei Harun Farocki). Zeitgleich beginnt die Medienkunst, sich mit den >anachronistischen< Medien Film und Fotografie auseinanderzusetzen und durch großflächige und multiple Projektionen des bewegten Bildes, welches als Videobild zunächst an den Fernseher gebunden ist, den Ausstellungsraum in die Komposition mit einzubeziehen. Die dominierenden Künstlerinnen und Künstler dieser Zeit sind selten auf ein bestimmtes Medium festzulegen, sondern nutzen verschiedene Ausdrucksformen, um die sie interessierenden Themen zu erarbeiten. Dabei lösen sie die klassische museale Präsentationsform des White Cube auf und »verwandeln die statischen Präsentationsbedingungen des Museums in einen Projektions- und Illusionsraum[...] «. ${ }^{6}$ Die Blackbox wird zu einem Gegenort der Ästhetik der Moderne, in dem die Betrachter/innen am Filmgeschehen teilnehmen können und wo der institutionelle und ästhetische Rahmen multiplen Projektions- und Erzählformen weicht: »Sie [die Blackbox, N. S.] bezieht ihre Kraft aus der Wiederbelebung einer Reizästhetik, die tendenziell auf die

2 Holger Kube Ventura, Politische Kunst Begriffe in den 199oer Jahren im deutschsprachigen Raum, Wien 2002, S. 65.

3 Ebd., S. 63.

4 Ebd.

5 Stefan Germer, »Kritik des Kanons. Was man von Gespenstern lernen kann« (1994), in: Germeriana: unveröffentlichte oder übersetzte Schriften von Stefan Germer zur zeitgenössischen und modernen Kunst, hg. v. J. Bernard, Jahresring 46, Köln 1999, S. 56-63, S. 6 o f.

6 Ursula Frohne, »That's the only now I get<. Immersion und Partizipation in VideoInstallationen«, in: Gregor Stemmrich (Hg.), Kunst/Kino, Köln 20o1, S. 217-238, S. 220. 
Immersionseffekte des Spektakels zurückgreift «. ${ }^{7}$ Ursula Frohne beschreibt zwei dominierende Tendenzen in der Film- und Videokunst hinsichtlich ihrer Bezugnahme auf das Kino: einerseits eine affirmative Reaktion auf kinematografische Illusions- und Wirkungsprinzipien, indem professionelle Kamera-, Ausstattungs-, Licht- und Schnitttechniken übernommen werden, um in perfekten Bild- und Soundinszenierungen hypnotische Wirkungen zu erzielen; ${ }^{8}$ andererseits eine Tendenz, Kino und Medienkultur als »konstruiertes Spektakel ${ }^{9}$ kritisch und mit konzeptuellem Ansatz darzustellen. Beispielsweise durch die Verwendung von Found Footage oder in Remakes werden filmische Repräsentationsmodelle hinterfragt und die Aufmerksamkeit auf die medialen Bedingungen der Darstellung und Wahrnehmung sowie auf das Dispositiv und die gegenüber musealen wie auch kinematografischen Arrangements veränderte Rolle der Betrachter/innen gelenkt. Der in der Beschreibung von Ausstellungsarchitekturen und Installationen vermehrt verwendete Begriff des Dispositivs geht im kinematografischen Kontext auf die psychoanalytische Filmtheorie der 1970er Jahre zurück. Er wurde durch JeanLouis Beaudry in zwei Aufsätzen ${ }^{10}$ geprägt und in der angloamerikanischen Rezeption durch Philip Rosen, Stephen Heath, Teresa de Lauretis und andere unter dem Stichwort des kinematografischen Apparats diskutiert.11 Grundgedanke der sogenannten Apparatustheorie ist die Auffassung, dass das Reale in der filmischen Repräsentation einem vielfachen Transformationsprozess unterliegt (durch Aufnahme, Schnitt, Vertonung, Projektion als Bewegtbild) und dass die Zuschauer/innen in ihrer immobilen Rezeptionshaltung zwischen Leinwand und unsichtbarem Projektor ideologischen Effekten

$7 \quad$ Ebd., S. 220 f. Zur Geschichte und Gegenwart der Blackbox in der Kunst vgl. auch die Aufsätze in Ralf Beil (Hg.), Black Box. Der Schwarzraum in der Kunst, Ausstellungskatalog Kunstmuseum Bern 2001, Ostfildern-Ruit 2001.

8 Frohne, »That's the only now I get ««, S. $222 \mathrm{f}$.

9 Ebd., S. 223.

10 Jean-Louis Baudry, »Ideologische Effekte erzeugt vom Basisapparat«, in: Robert Riesinger (Hg.), Der kinematographische Apparat. Geschichte und Gegenwart einer interdisziplinären Debatte, Münster 2003, S. 27-39, frz. Erstveröffentlichung als:»Cinéma. Effets idéologiques produits par l'appareil de base«, in: Cinéthique 7/8, 1970, S. 1-8; Ders., »Das Dispositiv. Metapsychologische Betrachtungen des Realitätseindrucks«, in: Riesinger, Der kinematographische Apparat, S. 41-62, frz. Erstveröffentlichung als: »Le dispositif. Approches métapsychologiques de l'impression de réalité«, in: Communications 23, 1975, S. 56-72.

11 Vgl. u. a. Teresa de Lauretis, Stephen Heath (Hg.), The Cinematic Apparatus, London/ Basingstoke 1980; Philip Rosen (Hg.), Narrative, Apparatus, Ideology. A Film Theory Reader, New York 1986. Der Begriff des Dispositivs in der Film- und Kinotheorie ist nicht gleichbedeutend mit der Bezeichnung systemischer und strategischer diskursiver Machtkonstellationen bei Foucault (vgl. dazu beispielsweise Giorgio Agamben, Was ist ein Dispositiv?, Zürich 2008 [ital. 2006]). 
ausgeliefert sind. Baudrys Modell, das sich jedoch nur auf den narrativen Spielfilm bezieht, wurde immer wieder herangezogen, um die veränderte Filmwahrnehmung durch Mehrfachprojektionen und die im Ausstellungsraum mobilen Betrachter/innen zu beschreiben. ${ }^{12}$

Ein neuer Bezug zur Performance und zum Körper im Film ist zu beobachten: die Künstler/innen rezipieren historische Vorbilder der 196oer und 197oer Jahre, grenzen sich dabei aber vom reduzierten, dokumentarischen Stil der frühen Videoarbeiten ab, indem beispielsweise »theatrale Mikrodramen « inszeniert werden, die die die verschiedenen Beziehungen zwischen Schauspieler/in, Zuschauer/in und potenziellem/r Mitspieler/in bewusst thematisieren. Darüber hinaus lassen die Möglichkeiten der Projektion in der Blackbox »kaleidoskopartige Panoramen aus Bewegungsbildern [entstehen], in denen die Protagonisten überlebensgroß agieren und in scheinbar leibhaftiger Präsenz den Raum wie eine Bühne bespielen «. $^{13}$ Ein weiterer Aspekt, der bei der eingangs beschriebenen Arbeit 24 Hour Psycho (1993) von Douglas Gordon und auch bei zahlreichen anderen Künstler/innen zum Tragen kommt, ist das Spiel mit der Verlaufszeit von Film, Video und projizierter Fotografie: Verlangsamung wird über technische Bearbeitung wie die Reduzierung der Abspielgeschwindigkeit erreicht oder aber durch die Verwendung langer Einstellungen und Montagen mit geringer Schnittfrequenz, die die Dynamik der Erzählung bremsen oder ganz zum Stillstand bringen (unter anderem bei Bill Viola, Tacita Dean, Mark Lewis, David Claerbout, Christoph Brech). Indem die Betrachter/innen mit ihren kinematografisch geprägten Sehgewohnheiten herausgefordert werden, sich langsamen oder quasi unbewegten Bewegungsbildern auszusetzen, verändern die Werke die Wahrnehmung von Filmzeit, erlebter Zeit und Dauer im Ausstellungskontext. Die Künstler/innen schließen damit an Experimentalfilme der 196oer und 1970er Jahre an, zum Beispiel von Michael Snow oder Chantal Akerman, die ebenfalls die erzählerische Wirkung der Montage zugunsten des bildlichen Potenzials des Films vernachlässigten.

Gregor Stemmrich stellt 2003 fest, dass eine Trennung der Bereiche Film und Kunst aus einer historischen Perspektive auf das 20. Jahrhundert wenig sinnvoll sei. Vielmehr lasse sich anhand der jeweiligen Erscheinungsorte und Präsentationformen die Tendenz eines Werks zum einen oder anderen Kontext

12 Vgl. u. a. Tanya Leighton, »Introduction«, in: dies.(Hg.), Art and the Moving Image. A Critical Reader, London 2008, S. 7-40; Katja Hoffmann, »Durch die Linse der Apparatustheorie. Ordnungen der Sichtbarkeit in Man Rays Experimentalfilm Les Mystères du Château du Dé (1929) und Eija-Liisa Ahtilas Videoinstallation The House«, in: Frohne, Haberer, Kinematographische Räume, S. 589-620.

Frohne, »>That's the only now I get ««, S. 224. 
feststellen. ${ }^{14}$ Die Kasseler documenta 5 (1972) etwa habe ein getrenntes, in einem Kino gezeigtes Filmprogramm präsentiert, das mit der eigentlichen Ausstellung wenig zu tun gehabt habe. Auch seien die Regisseure nicht im Katalog aufgeführt worden. Die Documenta1 im Jahr 2002 hingegen habe zahlreiche Formen der Filmpräsentation innerhalb der Ausstellungsräume gezeigt und sich einerseits bei den Konventionen des Kinos - abgedunkelte Räume und Sitzgelegenheiten -, andererseits bei denen des Ausstellungssettings bedient, indem die Besucher/innen einer Vorführung ohne feste Zeiten beiwohnen konnten. Dass die 199oer Jahre den Beginn eines Wandels in der gegenwärtigen Ausstellungspraxis bedeuten, begründet Erika Balsom mit der neuen Verfügbarkeit von hochauflösenden Videoprojektionen ab den späten 198oer Jahren. Sie ermöglichten für Balsom eine Loslösung von der beschränkten, monitorbasierten Präsentation, die in den vorherigen Jahrzehnten stärker an Skulptur, Malerei oder Performance orientiert war. Mit den technischen Möglichkeiten der großflächigen Projektion sei auch ein stärkerer Bezug auf kinematografische Konventionen wie Mise en scène, Montage, Spektakel, Narrativ und Illusionismus zu beobachten - ein Paradigmenwechsel innerhalb der Ausstellungskunst, wie Balsom schreibt. ${ }^{15}$ Die Ausstellung Passages de l'image 1990 im Centre Georges Pompidou, Paris, kann als Beispiel dafür dienen, wie Film und Video im Ausstellungsraum aufeinandertrafen. Passages de l'image kombinierte als erste Schau innovative Filmkunst von Jean-Luc Godard, Chris Marker, Michael Snow, Alexander Kluge und François Truffaut mit Videos und Fotografien von Gary Hill, Thierry Kuntzel und Jeff Wall. Die Ausstellung, schreiben die Kuratoren, sei dem Wunsch entsprungen, »zu verstehen, was sich zwischen den Bildern ereignet«. Es sei nicht mehr möglich, »wie früher von dem Kino, der Fotografie und der Malerei zu sprechen «, da sich das Bild in einer essenziellen Krise befinde, »die seine Natur selbst betreffe ${ }^{16}{ }^{16}$

Die diagnostizierte Krise des Bildes angesichts der vielfältigen medialen und ästhetischen Überschneidungen, Überlagerungen und Verschiebungen lenkt den Blick einerseits auf die Orte, an denen diese Art von Filmkunst

14 Gregor Stemmrich, »White Cube, Black Box and Grey Areas. Venues and Values«, in: Leighton, Art and The Moving Image, S. 430-443, S. 430.

15 Vgl. Balsom, Exhibiting Cinema in Contemporary Art, S. $12,18$.

16 »Cette exposition est née du désir de comprendre ce qui se passait dans les images et entre les images à partir du moment où il devenait clair qu'on ne pou-vait plus dire comme avant: le cinéma, la photo, la peinture. Parce qu'on était définitivement dans un temps de crise de l'image, touchant á la nature même des images.« (Raymond Bellour, Catherine David, Christine van Assche, »Préface«, in: Passages de l'image, Ausstellungskatalog Musée national d'art moderne, Centre Georges Pompidou, Paris, Fundació Caixa de Pensions, Barcelona, Power Plant, Toronto, Wexner Art Center, Columbus, Modern Art Museum, San Francisco 1990-1992, Paris 1990, S. 6-7, S. 6.). 
entsteht und eine Auseinandersetzung mit dem Dispositiv des Kinos und der Frage nach der Ausstellbarkeit bewegter Bilder virulent wird. ${ }^{17}$ Raymond Bellour spricht von einem sanderen Kino<, bei dem es schwierig sei, Grenzen und Begriffe abzustecken, da es gleichzeitig dem Dispositiv des Kinos wie auch der Tradition der Videokunst und der Filminstallation verwandt sei. ${ }^{18}$ Andererseits wird nach dem Zustand des Bildes selbst gefragt, das sich zwischen Fotografie, Video und Film ansiedelt und das sich nicht nur medial, sondern auch ontologisch in einem Zwischenraum befindet, weil Ästhetik, Materialität und Zeitlichkeit des Bildes nicht mehr klar und in etablierten Kategorien bestimmt werden können. Wiederum Raymond Bellour prägt dafür den Begriff »L'EntreImage «, 19 auf den noch zurückzukommen sein wird.

Die oben skizzierten Tendenzen der technischen und medialen Entgrenzung der Kunst sind seit den 196oer Jahren zu beobachten und erschweren die gattungstheoretische Einordnung der Werke. Sie wenden sich gegen eine modernistische Ästhetik des geschlossenen Kunstwerks und destabilisieren »gezielt die Grenzen zwischen den Künsten und zwischen Kunst und Nichtkunst «. ${ }^{20}$ Juliane Rebentisch spricht der zeitgenössischen Kunst kritisches Potenzial zu, da diese sich gegen die modernen Fortschrittsideen wende, zugleich aber eben nicht, wie vom Posthistoire propagiert, in einer Geschichtsvergessenheit verharre. Vielmehr plädiert sie dafür, die Gegenwartskunst als $»$ kritische Fortsetzung des modernen Projekts ${ }^{21} \mathrm{zu}$ verstehen, innerhalb derer die retrospektive Auseinandersetzung mit modernen Positionen und Ästhetiken als Ausdruck einer Gegenwartskunst im normativen Sinn zu verstehen sei, einer Kunst, die »ihre historische Gegenwart gegenwärtig machen soll «. ${ }^{22}$ Gerade die Entgrenzung aktueller Kunst, die sich gegen eine gattungslogische und traditionelle Einordnung und somit eine eindimensionale Lesbarkeit sträube, betone ihre Historizität und die Notwendigkeit, sie stets neu in ein Verhältnis zur Vergangenheit und zur jeweiligen Gegenwart zu setzen. ${ }^{23}$ Rebentisch nennt drei historische Zäsuren, die für die zeitgenössische Kunst

17 Vgl. hierzu unter anderem Frohne, »>That's the only now I get««, sowie Frohne, Haberer, Kinematographische Räume; Balsom, Exhibiting Cinema in Contemporary Art; Catherine Fowler, »Room for experiment: gallery films and vertical time from Maya Deren to Eija Liisa Ahtila«, in: Screen 45/4, 2004, S. 325-343; Dominique Païni, Le temps exposé. Le cinéma de la salle au musée, Paris 2002.

18 Raymond Bellour, »Über ein anderes Kino«, in: Stemmrich, Kunst/Kino, Köln 2001, S. 251-269.

19 Raymond Bellour, L'Entre-Images. Photo, Cinéma, Vidéo, Paris 2002 (199o).

20 Juliane Rebentisch, Theorien der Gegenwartskunst zur Einführung, Hamburg 2013, S. 21.

21 Ebd., S. 20.

22 Ebd., S. 13.

23 Vgl. ebd. 
wichtig seien: 1945, das Datum des Beginns einer Nachkriegsästhetik, die sich mit der Frage zu befassen hatte, inwiefern nach Auschwitz überhaupt noch Kunst möglich sei; 1965 als Schwelle, die von den Kuratoren der Tate Modern gesetzt wurde, um die allmähliche Öffnung und intermediale Entgrenzung der Künste in den 196oer Jahren zu markieren, und 1989 als weltpolitische Zäsur, die das Ende des Kalten Krieges und den Beginn der Globalisierung anzeigt, eine Zeit, in der sich durch Biennalen und Triennalen weltweit neue Zentren der Kunst bildeten und in der gleichzeitig durch die kritischen Fragen des Postkolonialismus die Verengung der Kunst auf die westliche Moderne angeprangert wurde. ${ }^{24}$

Wie an den einzelnen Beispielen zu sehen sein wird, spiegelt sich die Relevanz dieser zeithistorischen und politischen Zäsuren in vielen Werken wider, wenn auch oft nicht vordergründig thematisch in Form historischer Bezugnahmen, sondern mittelbar über die Reflexion historischer künstlerischer Tendenzen. Die Werke der hier im Fokus stehenden Künstlerinnen und Künstler weisen jeweils starke Bezüge zur Kunst der Moderne auf. Tacita Dean setzt sich in ihren Filmen beispielsweise mit Joseph Beuys, Marcel Broodthaers, Robert Smithson, Mario Merz, Giorgio Morandi, Claes Oldenburg und Cy Twombly auseinander. Die Kunst der Nachkriegszeit bis in die 1970er Jahre hinein wird bei ihr in ihrer eigenen Historizität dargestellt: der Darmstädter Werkblock von Beuys als Werk, das im Dialog mit dem eigens gestalteten Museumsraum langsam altert und Fragen nach Erhalt, Reflexion und Verfall moderner Werke stellt; die Ateliers von Twombly, Oldenburg und Morandi als Orte, in denen sich die historische Zeit an den bewahrten Dingen anlagert und von Deans Filmen wieder zum Leben erweckt wird. James Coleman hat als einer der ersten in den 1970er und 1980er Jahren das Fernsehmelodram und die Fotonovela für seine Foto-, Theater- und Videoarbeiten entdeckt und damit die Grenzen zwischen Hoch- und Populärkunst aufgeweicht. Mit seinen Diaprojektionen nahm er die Narration in Form der Bilderfolge und Korrespondenzen zwischen Fotografien zu einer Zeit in den Blick, als Fotografie als Medium der Konzeptkunst populär war. Mark Lewis' Filme zeigen einerseits modernistische Architekturen, die bereits wieder im Verfall begriffen sind, und reflektieren andererseits die frühe Filmästhetik vom Ende des 19. Jahrhunderts. Douglas Gordon schließlich lässt die Grenzen der Medien Film und Video in der Form der kinematografischen Installation verschwimmen, wenn er Spielfilme der Hollywoodära mittels Verlangsamung und (Mehrfach-)Projektion im Ausstellungsraum in einen Grenzbereich von Kino

24 Vgl. ebd., S. 14-19. Siehe auch den Band Alexander Dumbadze, Suzanne Hudson (Hg.),

Contemporary Art. 1989 to the Present, Chichester 2013. 
und Kunst führt. Die Zeitlichkeit des Bildes zeigt sich als das Moment, in dem die Überschreitung medialer und räumlicher Grenzen mit der Adressierung der Geschichtlichkeit der Dinge, Medien und Modi der Darstellung zusammenfällt.

\title{
Bild und Zeit in Kunstgeschichte, Film- und Medienwissenschaft
}

\section{Zum Verhältnis von Zeit und Geschichte}

In welchem Verhältnis stehen Zeit und Geschichte zueinander, und wie konkretisiert sich dieses im bewegten filmischen oder fotografischen Bild? Für Emil Angehrn sind Zeit und Geschichte nicht getrennt voneinander zu betrachten, denn sie stehen in einem unhintergehbaren Wechselverhältnis, das einerseits von Affinität, andererseits von Antithetik geprägt ist:

\begin{abstract}
Zeitlichkeit ist die allgemeine Struktur des Außereinanderseins-im-Nacheinander, Geschichtlichkeit das Eingefügtsein in einen konkreten Geschehensverlauf. Zeit ist eine bestimmte Form des Erlebens bzw. der Konstitution des Wirklichen, die etwa nach den beiden Grundrastern der Relationen von Früher-GleichzeitigSpäter und Vergangen-Gegenwärtig-Zukünftig strukturiert ist. ${ }^{25}$
\end{abstract}

Dieses »Formschema ${ }^{26}$ von Zeitlichkeit als Relationsverhältnis der unterschiedlichen Zeitebenen ist die Struktur und weltlich-anthropologische Konstante, in die der Mensch als historisches Wesen, das in der Zeit lebt und sich innerhalb dieser orientiert, eingefasst ist. Geschichte ist nach Angehrn der »konkrete, inhaltlich bestimmte Prozess« des menschlichen Daseins: »Der Mensch ist ausgespannt zwischen Gewesenem und Nochnichtseiendem, und nur indem er in irgendeiner Weise die Synthesis in diesem Zerstreutsein vollzieht, vermag er im Außersich mit sich eins zu sein. ${ }^{27}$

Die reine Zeitlichkeit als Spanne aller zeitlichen Möglichkeiten zwischen dem Vergangenen und dem Zukünftigen verlangt vom Menschen die Syntheseleistung, diese Bereiche ineinander zu vermitteln. Hinter dem, was Angehrn hier nur abstrakt beschreibt, verbergen sich Phänomene wie Erinnerung, phänomenologische Retention und Protention, Narration, Kategorien wie Augenblick und Dauer, Bewegung und Stillstand. Sie bilden die formale Basis für die konkrete historische Verankerung in der Welt, für die Definition von Zeitpunkten und Ereignissen, die sich ausschließlich an einer »bestimmten

25 Emil Angehrn, »Zeit und Geschichte«, in: ders., Der Sinn der Zeit, Weilerswist 2002, S. $67-84$, S. 69 .

26 Ebd., S. 70.

27 Ebd. 
Zeitstelle $\ll^{28}$ fixieren lassen, wie bereits Georg Simmel erklärte und Angehrn im Folgenden darstellt:

Darüber hinaus existiert der Mensch geschichtlich, ist er sich aus einer bestimmten Geschichte heraus gegeben, die ihn geprägt hat, in der sein Verstehen, sein Tun und Sein wurzelt, an der er teilhat und die er mitgestaltet. Die Formen zeitlicher Strukturierung und Synthetisierung sind ein Fundament seine[s] Geschichtlichseins, seiner Aneignung und Gestaltung der konkreten Geschichte, die darüber hinaus andere Formen historischer Konstitution einschließt: Formen der Darstellung und Interpretation, der theoretischen und praktischen Aufarbeitung, der Selbstverständigung über die Geschichte. Zeit und Geschichte benennen, so gesehen, unterschiedliche Konkretionsstufen: Mein geschichtliches Dasein ist die konkrete Art und Weise, wie ich in der Zeit bin und als zeitliches Wesen existiere. ${ }^{29}$

Die an künstlerischen Werken exemplarisch untersuchte Verzahnung von Zeit, Geschichte und Bild konkretisiert sich demnach in Aspekten wie der Wahrnehmung des Bildes, der zeitlichen Dauer, der Narrativität oder der Indexikalität als Parameter, die das bewegte Bild und seine zeitliche Figuration innerhalb des zeitlichen Rasters von Vergangenheit, Gegenwart und Zukunft verorten und ihm so Historizität verleihen. Weder kann ein Bild als aus seiner historischen Zeit herausgehoben betrachtet werden, noch kann (oder sollte) es als rein historisches Artefakt ohne Bezug zu den unterschiedlichen Zeitdimensionen beschrieben werden. ${ }^{30}$

Voraussetzung für diese Wahrnehmung von Geschichte als zeitlich und somit für die Beschreibung der Geschichtlichkeit eines Ereignisses ist die Verzeitlichung des Geschichtsbegriffs in der Moderne, wie sie besonders von Reinhart Koselleck beschrieben wurde. Wurden in vormoderner Zeit historische Ereignisse als unverrückbare Wahrheiten begriffen, die als Eckpunkte einer linearen Geschichtsschreibung dienten und, einmal abgeschlossen, maximal einen passiven Blick auf einen in sich abgeschlossenen Gegenstandsbereich erlaubten, so zeichnet sich das Geschichtsverständnis ab etwa Mitte des 18. Jahrhunderts durch einen perspektivischen Blick auf das Vergangene aus. Koselleck bezieht sich vor allem auf den Theologen und Historiker Johann Martin Chladenius (1710-1759), der den Begriff des Sehepunktes prägte: Demnach gibt es keine objektive historische Wahrheit, da alle Erfahrung

28 Vgl. Georg Simmel, »Das Problem der historischen Zeit« (1916), in: ders., Gesamtausgabe, hg. v. Otthein Rammstedt, Bd. 15, Frankfurt am Main 2003, S. 287-304, S. 294.

29 Angehrn, »Zeit und Geschichte«, S. 70.

30 Zeit ist nach Angehrn einerseits die »Tiefenstruktur der Geschichte«, andererseits sind die »spezifischen Zeitformen und Zeitbilder, die in unser historisches Verständnis eingehen, [...] ebenso von Geschichte(n) her gedacht« (ebd., S. 74). 
relativ ist und es somit auch einander wiedersprechende Augenzeugenberichte geben kann: »Denn es gibt einen Grund, warum wir die Sache so, und nicht anders erkennen: und dieses ist der Sehe-Punckt von derselben Sache ... Aus dem Begriff des Sehe-Puncts folget, daß Personen, die eine Sache aus verschiedenen Sehe-Puncten ansehen, auch verschiedene Vorstellungen von der Sache haben müssen... « «11 Diese zeitliche `Standortbindung desjenigen, der Geschichte erlebt, bezieht sich ebenso auf die Relativität des historischen Urteils. Die Erkenntnis setzte sich durch, dass die Vergangenheit sich durch die Zeitlichkeit des Blicks auf sie sehr wohl noch verändern kann, dass Geschichtlichkeit ein genuin zeitliches Phänomen ist. »Verzeitlichung der Geschichte« bedeutet also: Je mehr sich der Standort des Historikers / der Historikerin vom gegebenen Ereignis entfernt, desto stärker wird die Wahrnehmung der Geschichte zu einem zeitlichen Phänomen.

\section{Bild und Zeit in der Kunstgeschichte}

Die Zeit ist von Beginn der bildlichen Darstellung an ein immanenter Aspekt jedes Bildes, wenngleich die systematische Beschäftigung mit der Frage nach der Zeit im Bild erst in der zweiten Hälfte des 20. Jahrhundert einsetzte. Als einer der ersten formuliert der Kunsthistoriker Dagobert Frey 1955 das »Zeitproblem in der Bildkunst « ${ }^{32}$ und beschreibt darin inhaltliche, strukturelle, kompositorische und historische Aspekte der Zeit im Bild, wobei er sich vor allem auf Malerei bezieht. Frey kann hier deshalb als klassisches Beispiel für die kunsthistorische Forschung dienen, weil er die Zeitdarstellung und -erfahrung als Problem und nicht als Potenzial des Bildes versteht. Im Gegensatz dazu fragt Gottfried Boehm 1987: »Auf welche Weise konstituiert sich in jenem System der Darstellung, welches wir Bild nennen, die Erfahrung von Zeit? «33 Dass die Schwierigkeit, die Zeit überhaupt begrifflich zu fassen, ein Topos entsprechender Abhandlungen geworden ist, stellt Heinrich Theissing seiner Untersuchung Die Zeit im Bild voran. ${ }^{34}$ Um sich mit der Frage nach der Korrelation von Zeitlichkeit und Geschichtlichkeit in bewegten Bildern zeitgenössischer Werke fundiert beschäftigen zu können, erscheint

$31 \quad$ Johannes Martin Chladenius, Einleitung zur richtigen Auslegung vernünftiger Reden und Schriften, Düsseldorf 1969 (1742), S. 195, zit. n. Reinhart Koselleck u. a., »Geschichte, Historie«, in: Otto Brunner, Werner Conze, Reinhart Koselleck (Hg.), Geschichtliche Grundbegriffe 2, Stuttgart 1975, S. 593-717, hier S. 696.

32 Dagobert Frey, »Das Zeitproblem in der Bildkunst« (1955), in: ders., Bausteine zu einer Philosophie der Kunst, Darmstadt 1976, S. 212-235.

33 Gottfried Boehm, »Bild und Zeit«, in: Hannelore Paflik-Huber (Hg.), Das Phänomen Zeit in Kunst und Wissenschaft, Weinheim 1987, S. 1-23, S. 2.

Heinrich Theissing, Die Zeit im Bild, Darmstadt 1987, S. 8. 
es ratsam, die wichtigsten historischen Positionen und Antworten darauf zu rekapitulieren und mit Ansätzen aus den Film- und Medienwissenschaften zu vergleichen. Dabei interessiert im folgenden Kapitel nicht die Zeit als unbestimmbares »Etwas « ${ }^{35}$, sondern Zeitlichkeit als Eigenschaft des Bildes, die durch Bewegung und Stillstellung, durch die Erfahrung von Augenblick und Dauer, durch Erzählstrukturen, durch bildimmanente Relationen und die Wahrnehmung des Betrachters / der Betrachterin ansichtig wird.

Als wichtigste Theorie über die ästhetische Betrachtung der Zeit in der Kunst seit der Aufklärung gilt in der Kunstgeschichte nach wie vor der von Gotthold Ephraim Lessing 1766 in seiner Schrift »Laokoon: oder über die Grenzen der Malerei und Poesie ${ }^{36}$ formulierte Gedanke einer Unterscheidung von Raum- und Zeitkünsten. Lessing verfolgt darin zwei Intentionen, wie Ingrid Kreuzer in ihrem Nachwort zusammenfasst:

Die eine - gegen eine Tradition gerichtet - strebt höchst wirksam die Befreiung der Poesie vom Diktat der Malerei an, das in der Formel >ut pictura poiesis $<u$ orthodoxer Gültigkeit erstarrt war. Die andere - ausgelöst von einem aktuellen Tatbestand - zielt auf die Begründung des Primats der Dichtkunst vor der Plastik $[\ldots] .{ }^{37}$

Als Reaktion auf Johann Joachim Winckelmanns kurz vorher erschienene Gedancken über die Nachahmung der Griechischen Werke in der Mahlerey und Bildhauer-Kunst, deren Idee der »edlen Einfalt« und »stillen Grösse« der römischen Laokoongruppe ihm widerstrebte, stellte Lessing in seiner Betrachtung der Gruppe den »fruchtbaren Augenblick« von Skulpturen und Gemälden in den Mittelpunkt. ${ }^{38}$ Dieser besondere Augenblick sollte es für die Betrachtenden möglich und notwendig machen, das Vorher und Nachher des dargestellten Moments zu imaginieren und so an der Wirkung des Kunstwerks aktiv teilzuhaben: »Dasjenige aber nur allein ist fruchtbar, was

35 Ebd., S. 13.

36 Gotthold Ephraim Lessing, »Laokoon: oder über die Grenzen der Malerei und Poesie«, Erster Teil, in: ders., Werke und Briefe, hg. v. Wilfried Barner, Bd. 5.2: Werke 1766-1769, Frankfurt am Main 199o, S. 11-206.

37 Ingrid Kreuzer, »Nachwort«, in: Gotthold Ephraim Lessing, Laokoon, oder Über die Grenzen der Malerei und Poesie. Mit beiläufigen Erläuterungen verschiedener Punkte der alten Kunstgeschichte (1766), Stuttgart 2006, S. 215-230, S. 219.

38 Wenngleich Lessings Schrift noch heute zum Fixpunkt ästhetischer Debatten über die Künste zählt, ist sie doch nicht originär: Seine Scheidung der Künste und die Heraushebung des punctum temporis finden sich zuvor bereits bei Shaftesbury, Diderot und Mendelssohn (siehe Inka Mülder-Bach, »Bild und Bewegung. Zur Illusion bildnerischer Bewegung in Lessings Laokoon«, in: Deutsche Vierteljahrsschrift für Literaturwissenschaft und Geistesgeschichte 66, 1992, S. 1-30, S. 17). 
der Einbildungskraft freies Spiel lässt. « ${ }^{39}$ Lessing unterscheidet zwischen Malerei und Poesie aufgrund ihrer materialen Beschaffenheit und aufgrund der jeweiligen Relation zu Raum und Zeit. ${ }^{40}$ Malerei und Bildhauerei bestünden als Körper aus »nebeneinander geordneten Zeichen «, die nur Gegenstände - wenn möglich im prägnantesten Moment der darzustellenden Handlung - zeigen könnten; die Poesie könne mittels ihrer »aufeinanderfolgenden Zeichen « Handlungen darstellen, dies aber nur beschränkt über die Schilderung von Körpern. ${ }^{41}$ Kreuzer stellt heraus, dass der »Zeitbegriff [...] in Lessings Theorie die zentrale Rolle [spielt] «. ${ }^{42}$ Seine starre Trennung von sogenannten Raumkünsten (unter die er die bildende Kunst im Allgemeinen, nicht nur die Malerei, fasst) und Zeitkünsten wie der Musik oder der Dichtung sei nur oberflächlich betrachtet tatsächlich durchgehalten, da er auch die Raumkünste als zeitliche denke, Raum sei für ihn »wesentlich zum Stillstand gebrachte Zeit «. ${ }^{43}$

Lessings »Laokoon « rief in der ästhetischen Debatte um den Paragone zahlreiche, auch kritische Reaktionen hervor, unter anderem von Herder und Goethe. ${ }^{44}$ Für die hier verfolgte Fragestellung ist die Debatte um die Raumund Zeitkünste insofern interessant, als Zeitlichkeit dort bestimmten Künsten aufgrund ihrer Materialität zugeschrieben wird und nicht als ästhetisches Moment dem Bild an sich eigen ist. Diese Argumentation wird sich, wie wir später sehen werden, im 19. und 20. Jahrhundert im Zuge der Erfindung von Fotografie und Film und der Diskussion um die jeweilige Zeitlichkeit des Mediums wiederholen. Dass Lessing Raum und Zeit als getrennte Größen behandelt, ist in der Kunstgeschichte weithin kritisiert worden, wie Inka Mülder-Bach ausführt, sie weist jedoch darauf hin, dass durch diese verengende

39 Lessing, »Laokoon: oder über die Grenzen der Malerei und Poesie«, S. 32. Vgl. hierzu auch Oskar Bätschmann, »Lessing installiert den fruchtbaren Betrachter«, in: Thomas Kisser (Hg.), Bild und Zeit. Temporalität in Kunst und Kunsttheorie seit 180o, München 2011, S. 21-48.

40 Vgl. Kreuzer, »Nachwort «, S. 221.

41 Vgl. Lessing, »Laokoon: oder über die Grenzen der Malerei und Poesie«, S. 116 f.

42 Kreuzer, »Nachwort«, S. 221.

43 Ebd.

44 Johann Gottfried Herder, »Die Kritischen Wälder zur Ästhetik. Erstes Wäldchen« (1769), in: ders., Schriften zur Ästhetik und Literatur $1767-1781$, hg. v. Günther E. Grimm, Frankfurt am Main 1993, S. 63-245, S. 131; Johann Wolfgang von Goethe, »Über Laokoon«, in: Helmut Pfotenhauer, Peter Sprengel (Hg.), Klassik und Klassizismus, Frankfurt am Main 1995, S. 149-161. Zur Auseinandersetzung mit dem »fruchtbaren Augenblick« siehe auch Ernst H. Gombrich, »Der fruchtbare Moment. Vom Zeitelement in der bildenden Kunst«, in: ders. (Hg.), Bild und Auge. Neue Studien zur Psychologie der bildlichen Darstellung, Stuttgart 1984, S. 40-61. 
Kritik die »fruchtbare Pointe « von Lessings Text verfehlt werde. ${ }^{45}$ Zwar ist er in seiner semiotischen Auslegung der Künste strikt, er stellt jedoch auch fest: »Doch alle Körper existieren nicht allein in dem Raume, sondern auch in der Zeit «. ${ }^{46}$ Mülder-Bach folgert:

Lessing leugnet nicht, daß sich eine Bewegungsspur in das Bild einzeichnen läßt; seine Theorie des >fruchtbaren Augenblicks ist im Gegenteil darauf angelegt, die Einbildungskraft auf diese Spur zu bringen. Was er zunächst leugnet, ist, daß Bewegung von der bildenden Kunst auf >natürliche< Weise bezeichnet werden kann. ${ }^{47}$

Zwar könne nach Lessing ein Körper eine Handlung nur andeuten und niemals tatsächlich ausführen, jedoch erlaubten die Darstellungs- und Ausdrucksmittel der Kunst in dieser Andeutung, die Einbildungskraft des/der Betrachters/in in Gang zu setzen und so dem Körper seine zeitliche Dimension wiederzugeben..$^{48}$

In seinem Aufsatz »Das Zeitproblem in der Bildkunst « beschreibt Dagobert Frey ausgehend von dem Problem der Darstellung von Zeit im statischen Bild verschiedene Aspekte der Zeitlichkeit des Bildes. Dem Werk der bildenden Kunst spricht er zu Beginn »objektive Unveränderlichkeit « und »Dauer ${ }^{49}$ sowie einen »überzeitlichen Charakter ${ }^{50} \mathrm{zu}$. Bildnerische Werke erhielten einen »Ewigkeitswert «, ${ }^{51}$ wodurch umgekehrt der Verfall den Aspekt der Vergänglichkeit betone. Da dem Kunstwerk Überzeitlichkeit eigen sei, stehe es notwendigerweise in einem Spannungsverhältnis zur individuellen Zeitlichkeit der Betrachtenden und deren Umwelt. ${ }^{52}$ Frey beschreibt, dass sich eine Wechselwirkung zwischen der Lebenswelt des/der Betrachters/in und der »überzeitlichen Realitätssphäre des Bildwerks« ergebe, wodurch auch

Mülder-Bach, »Bild und Bewegung «, S. 18.

Lessing, »Laokoon: oder über die Grenzen der Malerei und Poesie«, S. 116.

Mülder-Bach, »Bild und Bewegung«, S. 18.

Vgl. ebd. Jüngst wiesen u. a. Oskar Bätschmann und Johannes Grave darauf hin, dass die Kritik an Lessing vielfach übersehe, dass er selbst die zeitliche Dimension der sogenannten Raumkünste keineswegs verneinte, sondern die Temporalität der Betrachtung eines Werks konkret benannte. Vgl. Bätschmann, »Lessing installiert den fruchtbaren Betrachter«, S. 29, und Johannes Grave, »Der Akt des Bildbetrachtens. Überlegungen zur rezeptionsästhetischen Temporalität des Bildes«, in: Michael Gamper (Hg.), Zeit der Darstellung. Ästhetische Eigenzeiten in Kunst, Literatur und Wissenschaft, Hannover 2014, S. 51-71, S. 51.

Frey, »Das Zeitproblem in der Bildkunst«, S. 212.

Ebd., S. 213.

Ebd.

Ebd., S. 214. 
das »zeitlich Distanzierte« in der Betrachtung »vergegenwärtigt« werde. ${ }^{53}$ Interessant für unsere Fragestellung ist Freys Feststellung, dass für das Kunstwerk trotz seiner herausgehobenen Zeitlichkeit die geschichtliche Komponente eine Rolle spielt. Dies sei nicht nur für die Forschung wichtig, denn »die Kunstgeschichte als historische Disziplin betrachtet grundsätzlich das Kunstwerk als geschichtliches Phänomen «.54 Vielmehr fragt er, »inwieweit in der Gestaltung des Bildwerkes selbst wesenhaft und intendiert die geschichtliche Entstehung und damit seine geschichtlich-zeitliche Festlegung zum Ausdruck gelangt«, 55 also welche Rolle die historische Persönlichkeit des Künstlers oder der Künstlerin wie auch die Entstehungszeit spielten. Für die Rolle der Zeit im Bild gelte dann: »Als persönliche Schöpfung ist das Kunstwerk zeitgebunden, und die geschichtliche Zeit wird in ihm anschaulich, für die rein ästhetische Betrachtung ist es überzeitlich. «56 Frey verknüpft die Frage nach der Geschichte ausschließlich mit dem Entstehungsprozess des Werks und unterscheidet sie von der Erzählung, die als »epischer Zeitablauf « grundsätzlich eine einseitige, nicht umkehrbare Richtung habe. ${ }^{57}$ Diese werde im Bild durch die Ausrichtung der Figuren frontal oder im Profil deutlich, wobei durch Letzteres auch verschiedene räumliche Ebenen des Bildes miteinander verbunden würden. Die Erzählung erfolge beispielsweise in Friesen oder auf Teppichen, indem Bildzeichen aneinandergereiht und nacheinander abgelesen würden, die Bildbetrachtung erfolge sukzessive. ${ }^{58}$ Jedoch biete ein Bild neben der sukzessiven Bilderschließung natürlich auch die simultane Wahrnehmung aller Bildelemente, den Überblick über Komposition und Aufbau des Werks: »Bei einem solchen Überblick wird sich ein bestimmter Rhythmus, werden sich Verdichtungen und Zäsuren, kürzere oder längere bildmäßig zusammengehörige Abschnitte, zusammenhängende Bewegungszüge und Ruhepunkte etwa in Frontalfiguren oder symmetrisch komponierten Gruppen zeigen. $\aleph^{59}$ Die Komposition eines Bildes durch die Anordnung von Figuren,

53 Ebd., S. 215 .

54 Ebd., S. 216.

55 Ebd.

56 Ebd., S. 218.

57 Ebd., S. 223.

58 Ebd., S. 224.

59 Ebd., S. 225. An dieser Stelle verweist Frey auf Franz Wickhoff, der die Beschreibung einer Bilderzählung in einem einzelnen Bild, hier innerhalb der Buchmalerei der Wiener Genesis, die Zusammenfassung mehrerer Zeitphasen eines Handlungsablaufs durch wiederholtes Auftreten derselben Person als »continuierende Darstellungsart« bezeichnet hatte (ebd., siehe Wilhelm Ritter von Hartel, Franz Wickhoff [Hg.], Die Wiener Genesis, 2 Bde., Jahrbuch der kunsthistorischen Sammlungen des Allerhöchsten Kaiserhauses, Beilage zum 15. und 16. Bd., Prag/Wien 1895, S. 8). 
Flächen und Objekten ist für filmische oder fotografische Werke gleichermaßen von Bedeutung. Insbesondere Filme, die durch die Länge und Langsamkeit der einzelnen Einstellungen den Blick des/der Betrachters/in nicht durch innerbildliche, handlungsorientierte Bewegungen lenken, sondern es ermöglichen, ihn schweifen zu lassen, erzielen ihre Wirkung nicht unwesentlich durch die Wahl des Ausschnitts und die Anordnung der Elemente im Bildfeld.

Schließlich geht Frey auf die Darstellung von Bewegung in der Bildkunst ein: »Das Zeitproblem zeigt sich in der Bildkunst in aller Eindringlichkeit dort, wo eine Bewegung im unbeweglichen, unveränderlichen Bild zur Darstellung kommt. Hier tritt die Spannung zwischen Überzeitlichkeit der Darstellung und Zeitlichkeit des Dargestellten in schärfster Form in Erscheinung. «60 Frey unterscheidet drei Möglichkeiten der Verbildlichung von Bewegung: 1. durch ein Bildzeichen oder Ideogramm, also die schematische Darstellung beispielsweise des Laufens in Höhlenmalereien, 2. durch die Heraushebung eines charakteristischen Augenblicksbildes aus dem Bewegungslauf - diesem schreibt Frey ähnlich wie Lessing zeitliche Fülle zu, indem darin das Vorher und Nachher mit umfasst werden können, und 3. durch die Zusammenfassung verschiedener Bewegungsphasen, wofür er die griechische Vasenmalerei ebenso als Beispiel anführt wie die »Synthese verschiedener Stellungen eines Gesichts« im Kubismus. ${ }^{61}$ Bewegung könne aber auch im rein Formalen ausgedrückt werden, er nennt hier das Linienornament, das im Nachvollzug als kinästhetisches Erlebnis durch die Betrachtenden als zeitlich erfahren werde: Wenn Bewegung das ganze Bild erfasse, liege »[d]as Unveränderliche $[. .$. nicht in der Statik, sondern der Dynamik des Bildes «.62

Zwar entstand Freys Text erst 1955, zeitlich bewegte Bilder wie die des Films fanden jedoch keinen Eingang in seine überblicksartige Untersuchung. Dennoch spielen einige der genannten Aspekte auch für diese eine Rolle. So lassen sich formale Bewegungsmomente auch am zeitlich bewegten Bild beschreiben, und ebenso wie eine das ganze Bild erfassende Dynamik, wie sie beispielsweise im Experimentalfilm häufig zu finden ist, spielt das Phänomen der Dauer und Zeitdehnung eine Rolle als »sich Veränderndes [...], an dessen gleichmäßigem, langsamen, schleichenden Ablauf die Zeit bewußt wird «.63 Insbesondere die starke Betonung der Erfahrung durch den/die Betrachter/ in und die zeitliche Verknüpfung von gegenwärtiger Bildbetrachtung und historischer Dimension des Kunstwerks wird bei der Analyse von Filmarbeiten

6o Frey, »Das Zeitproblem in der Bildkunst«, S. 221.

61 Vgl. ebd., S. $221 \mathrm{f}$.

62 Ebd., S. $230 \mathrm{f}$.

63 Ebd., S. 220. 
eine Rolle spielen, beispielsweise bei Tacita Dean, die den handwerklichen Aspekt ihrer Werke stets betont. Als problematisch erweist sich jedoch Freys dualistisches Zeitverständnis, das aus seiner apodiktischen Setzung des Kunstwerks als »überzeitlich« resultiert, wobei er von einem normativen Kunstbegriff ausgeht, der das Werk primär auf seine religiöse und soziale Funktion reduziert. ${ }^{64}$ Die Gegenüberstellung von ästhetischer Überzeitlichkeit einerseits und in der Betrachtung vergegenwärtigter Erfahrung des primär gegenständlichen Bildes andererseits bindet die Zeit an benennbare Motive und Bildinhalte, verkennt aber eine strukturelle Dimension der Zeitlichkeit im Bild, welche das Verhältnis von Bildelementen, Bildfeld und Bildbetrachtung als ein genuin zeitliches definiert (hierzu siehe weiter unten den Ansatz von Gottfried Boehm). Bewegung zeigt sich für Frey vor allem in Motiven, in der räumlichen Komposition des Bildes und in der Einfühlung der Betrachter/ innen in die Dynamik abstrakter Formen; es ist mittels seiner Lesart jedoch nicht möglich, beispielsweise Aspekte wie die Prozessualität einer Figur im Bild, die Zeitlichkeit bestimmter Medien und Materialien und ihres Realitätsbezugs oder das Verhältnis korrespondierender Bildelemente in der Collage oder Montage zueinander zu fassen. Freys Verständnis von Historizität bezieht in keiner Weise die Erinnerung und Imagination der Betrachtenden mit ein, ihre Möglichkeit zur Verknüpfung bildimmanenter Verweise mit einem individuellen Bildgedächtnis oder die einem Medium wie dem Film innewohnende Überlagerung zeitlicher Abläufe und historischer Verweise.

In zwei Aufsätzen widmet sich Lorenz Dittmann der Zeitstruktur beziehungsweise Zeitgestalt des Gemäldes und somit nicht einer allgemeinen Bestimmung der Bedeutung der Zeit für die Kunst, sondern der konkreten, in der Anschauung des Werks virulenten Rolle der Zeit. ${ }^{65}$ So unterscheidet er 1. die geschichtliche Zeit eines Werks, die durch Datierung, Zuordnung, Ikonografie und Stilgeschichte bestimmt wird, 2. die dargestellte Zeitsituation, das heißt die Bilderzählung oder dargestellte Handlungsabläufe, und 3. die dem künstlerischen Aufbau selbst inhärente Zeitlichkeit. ${ }^{66}$ Letztere entsteht aus

64 Vgl. ebd., S. 232.

65 Lorenz Dittmann, »Über das Verhältnis von Zeitstruktur und Farbgestaltung in Werken der Malerei«, in: Friedrich Piel, Jörg Traeger (Hg.), Festschrift Wolfgang Braunfels, Tübingen 1977, S. 93-109; ders., »Überlegungen und Beobachtungen zur Zeitgestalt des Gemäldes«, in: Neue Hefte für Philosophie 18/19, 1980, S. 133-150.

66 Dittmann, »Überlegungen und Beobachtungen zur Zeitgestalt des Gemäldes«, S. 133. Dittmann bezieht sich in seiner Unterscheidung verschiedener Zeitaspekte auf Etienne Souriau, der bereits von »intrinsic time of the work of art « sprach (vgl. Etienne Souriau, »Time in the Plastic Arts«, in: The Journal of Aesthetics and Art Criticism 7/4, Juni 1949, S. 294-307, S. 297). Auch Souriau orientiert sich an einer Zweiteilung der Künste in Raumund Zeitkünste, kritisiert aber eine Theorie des fruchtbaren Augenblicks der Raumkünste 
dem Zusammenwirken von Bildaufbau und Wahrnehmung des Bildes und wird von Dittmann als »Zeitgestalt « bezeichnet: »Die Bildgestalt bestimmt, als Zeitgestalt, je neu den Charakter der Zeitlichkeit.« ${ }^{67}$ Dittmann orientiert sich an Kurt Badts Feststellung, dass die Folgeordnung - die schematische, räumliche Anordnung von Bildelementen - eine unmittelbar zeitliche Komponente hat, und zwar im Akt der Wahrnehmung durch den/die Betrachter/in. Anhand verschiedener Beispiele beschreibt Dittmann, wie sich in der Zeitgestalt im Bild Vergangenheit und Zukunft in der Gegenwart der Betrachtung verbinden. Ebenso wie Frey geht er also von einer zunächst räumlichen Ordnung des Bildes aus, aus der eine zeitliche Dimension resultiert.

Heinrich Theissing unterscheidet ebenso wie Dittmann drei Zeitaspekte in Kunstwerken: »die historische Zeit, in der das Werk existiert, die Betrachtungszeit, in der es erfahren wird, und die dem Werk eigene Zeit - die Bildzeit, welche durch die >dargestellten Zeitsituationen« und mehr noch durch ihre zeitliche Darstellungsweise zur Anschauung kommt «.68 Die historische Zeit betrifft die Einbettung des Kunstwerks in seine Epoche und die spezifischen historischen Bedingungen seiner Entstehung. Jedes Werk sei ein »Erzeugnis einer geschichtlichen Persönlichkeit, die ihrerseits eine Geschichte hat; und es ist durch die Geschichte selbst und den Geist seiner Zeit geprägt«;69 die künstlerische Formensprache, Stil und Ausdruck, Material und Technik, Thematik und zeitgenössische oder historische Referenzen sind demnach Teil dieser spezifischen Historizität eines jeden Werks. Es gehört nach Theissing zu den elementaren Aufgaben der Kunstgeschichte, die historische Zeit eines Werks zum Ausgangspunkt für die Analyse und Interpretation zu nehmen und es biografisch, geschichtlich und geistesgeschichtlich einzuordnen.70 Die historische Zeit beinhalte darüber hinaus die im Bild angelegten Verknüpfungen zwischen der historischen Zeit des Bildes und einer symbolhaften Überzeitlichkeit, beispielsweise bei Ikonen, und intertextuelle Zitate innerhalb der eigenen bildnerischen Tradition durch das Zitieren eines Stils. ${ }^{71}$ Theissing stellt dem die Betrachtungszeit gegenüber und konstatiert zunächst, dass Lessings Trennung von Raum- und Zeitkünsten ein »Irrtum « sei und auch die bildenden Künste Malerei und Skulptur sehr wohl Zeit, nämlich die Zeit der Aufmerksamkeit und Betrachtung erforderten. Mit Verweis auf die

als falsch. Interessanterweise erwähnt Souriau als einziger auch das Kino als eine Art der Zeitkünste.

67 Dittmann, »Überlegungen und Beobachtungen zur Zeitgestalt des Gemäldes«, S. 135.

68 Theissing, Die Zeit im Bild, S. 18.

69 Ebd.

70 Vgl. ebd.

71 Vgl. ebd., S. 23 f. 
Erkenntnisse der Wahrnehmungspsychologie im 2o. Jahrhundert - er bezieht sich hier insbesondere auf Arnheim und Gombrich - beschreibt Theissing, wie die Betrachtung von Bildwerken durch das Tasten des Auges stets aktiv ist, kein »passives Empfangen« sondern »Sehakt« (Arnheim). ${ }^{72}$

Die sogenannte Bildzeit nimmt vorhersehbar den größten Teil von Theissings Untersuchung ein, auch weil die historische Zeit und die Zeit der Wahrnehmung Eingang in sie finden. Die Bildzeit ist die »imaginäre « Zeit des Bildes, sie betrifft die Frage, »wie in seiner Formensprache ein bestimmter Zeitsinn sich mitteilt «.73 Theissing beobachtet zwei Paradoxien: die »Unsichtbarkeit der Zeit und de[n] Stillstand eines Geschehens im Bilde « ${ }^{74}$ Erstere entlarvt er als Scheinproblem, da nicht nur in der Kunst, sondern auch in Wirklichkeit Zeit nicht sichtbar ist. Sie muss über die Sinne erfahren werden, womit Theissing einen grundsätzlich phänomenologischen Zugang wählt. Ähnlich wie Boehm rückt er die Erfahrung der Zeitlichkeit in den Vordergrund. Seine Diskussion des Stillstands des Geschehens verweist darauf, dass Bilder generell nicht bewegt seien. Dies stehe im Widerspruch zu der bei Aristoteles formulierten Erkenntnis, dass Zeit »etwas wesenhaft Kinetisches « ${ }^{75}$ sei - Zeit sei demnach nicht ohne Bewegung zu bestimmen und Bewegung nicht ohne Zeit. ${ }^{76}$ Die Bewegung bei Aristoteles als kinēsis betreffe aber nicht nur die Ortsveränderung, die ja in statischen Bildwerken nicht vorkomme, sondern allgemein auch die Veränderung von Eigenschaften, die mit den Mitteln der Malerei darzustellen seien, daher erledige sich das Problem, dass Bewegung im Bild nur suggeriert werden könne: »Ihre Gegenwärtigkeit im Jetzt, welche sie aus der Zeit hebt, enthält doch anschauliche Veränderungen und somit Zeit.«77 Theissing subsumiert:

Die Zeit ist nicht sichtbar, aber sie wird sichtbar an den Erscheinungen im Bild durch Qualitätsveränderungen >von etwas her zu etwas hin<, Veränderungen, welche die Ausdehnung, die Intensität, den Wandel und das Hervortreten und Verschwinden der Elemente betreffen, die den anschaulichen Bildgehalt fundieren. ${ }^{78}$

\footnotetext{
72 Ebd., S. 27.

73 Ebd., S. 34 .

74 Ebd., S. 35 .

75 Ebd., S. 41.

76 Vgl. Aristoteles, Physik. Vorlesungen über Natur, Erster Halbband: Bücher I(A)-IV( $(\Delta)$, übersetzt, mit einer Einleitung und mit Anmerkungen hg. v. Hans Günter Zekl, Hamburg 1987, S. 219.

77 Theissing, Die Zeit im Bild, S. 42.

78 Ebd.
} 
Zur Bestimmung der Bildzeit beruft sich Theissing auf die klassischen Strukturelemente der kunsthistorischen Bildbeschreibung »Umriß«, Ebene«, »Raum« und $»$ Farbe $« .79$ Insbesondere in der Formenanalyse und der Gestaltpsychologie Arnheims sind diese Kategorien als Leitpunkte der Strukturanalyse zu finden, und Theissing verwendet sie, um die Bewegungsmotive mit Blick auf ihre Zeitlichkeit zu analysieren.

Wo Frey, Dittmann und Theissing von der klassischen Malerei ausgehen und die Darstellung von Zeit als Problem des statischen Bildes behandeln, betrachtet George Kubler 1962 die Kunst selbst als zeitliches Phänomen, deren Entwicklung er als Problemgeschichte ${ }^{80}$ begreift. In seinem Essay The Shape of Time entwirft er eine Kunstgeschichte, die sich nicht an klassischen Zugängen zum Werk wie der Künstlerbiografie, der Kunstsoziologie, der Ikonografie oder der Stilgeschichte orientiert, sondern fragt, wie sich ein Artefakt durch seine spezifischen Formen auszeichnet und in die Zeit einordnen lässt. Kubler fasst damit erstmals nicht nur Kunstwerke im eurozentrierten Sinn der Gattungen, sondern »alle von Menschen geschaffenen Dinge, einschließlich aller Werkzeuge und alles Geschriebenen ${ }^{81}$ unter die Idee der Kunst. Unter dem »Oberbegriff der visuellen Form« diene die Geschichte der Dinge dazu,

Ideen und Gegenstände [...] wieder zu vereinigen: der Terminus beinhaltet [...] kurz gesagt, alle Arten von Material, die von Menschenhand bearbeitet worden sind, geleitet von verbindenden Ideen, die sich im Laufe einer zeitlichen Sequenz entwickelt haben. Aus allen diesen Dingen läßt sich die Form einer Zeit ablesen. ${ }^{82}$

Kubler wendet sich von der Kategorie des Stils und seinen »biologischen und physikalischen Metaphern « als Erklärungsmodell einer kunstgeschichtlichen Entwicklung ab. ${ }^{83}$ Vielmehr stellten die Artefakte die jeweils aktuellen Lösungen für ein künstlerisches, formales oder technisches Problem dar, für das es bereits vorher und auch später andere Lösungsansätze gegeben hat beziehungsweise geben wird. Die zeitliche Relation verschiedener Lösungen zueinander ist hier die Sequenz (im Gegensatz zur Epoche oder Periode), so dass der Ausdruck »Lösungsketten« den historischen kausalen Zusammenhang von

79 Theissing widmet jeder der Kategorien ein eigenes Kapitel.

8o $» J e d e s$ bedeutende Kunstwerk kann als ein historisches Ergebnis angesehen werden und als die schwer erarbeitete Lösung eines Problems. « (George Kubler, Die Form der Zeit. Anmerkungen zur Geschichte der Dinge, übersetzt von Bettina Blumenberg, mit einer Einleitung von Gottfried Boehm, Frankfurt am Main 1982 [1962], S. 71.)

81 Ebd., S. 32.

82 Ebd., S. 42.

83 Ebd., S. $40 \mathrm{f}$. 
Werkgruppen oder -folgen bezeichnet. Entwicklungen können anhand dieses Modells auch über längere Zeiträume und Regionen beobachtet werden, sie können diskontinuierlich sein und auch Unterbrechungen aufweisen. Kubler vertritt daher eine nichtlineare Konzeption von Geschichte und Zeitlichkeit. Vergangene Ereignisse würden nur über Signale übermittelt und im >Jetzt< wahrgenommen, unmittelbar erfassen könnten wir nur die »Aktualität«. ${ }^{84}$ Gottfried Boehm fasst Kublers Zeitverständnis zusammen:

Kubler entwirft ein strukturelles Verständnis der Zeit und des historischen Prozesses nicht vom Zeitinhalt her, sondern vom >Wesen der Aktualität $<$. An der Gegenwart von Zeit wird deutlich, daß es - in seiner Sicht - letztlich ein leeres Intervall ist, das einerseits das Zukünftige vom Vergangenen trennt. Dieser leere Augenblick ist andererseits aber auch alles, was wir je unmittelbar erfahren können. Insofern gliedert das leere Intervall die Zeit, schafft Relationen, macht sie meßbar und verleiht ihr eine historische Dimension. ${ }^{85}$

Boehm befasst sich in seiner Einleitung mit Kublers methodischem Problem, Kunst und Geschichte zu einer Kunstgeschichte zu verbinden. Die Aufgabe des Historikers sei nach Kubler die »Darstellung von Zeit«:86

Zeit als solche ist - wie Bewußtsein - nicht erkennbar. Wir kennen Zeit nur indirekt durch das, was in ihr geschieht: durch die Beobachtung von Dauer und Wandel; durch die Abfolge von Ereignissen und die dazwischenliegenden Phasen der Unveränderlichkeit; und indem wir unterschiedlich lange Zeitabstände zwischen den Veränderungen wahrnehmen. ${ }^{87}$

In seiner Theorie versuche Kubler, so Boehm, ebenso wie zuvor die Stilgeschichte eine Verknüpfung der Würdigung und historischen Alleinstellung des einzelnen Kunstwerks mit der Historisierung und Vernetzung mit anderen Objekten. Kublers Theorie wurde nach Erscheinen vor allem über die Fachgrenzen hinaus wahrgenommen. Starken Einfluss hatte sie auf eine Reihe von Künstlern, unter anderem Robert Smithson, mit dessen Land-Art-Skulptur Spiral Jetty sich Tacita Dean in ihrem Film JG (2013) intensiv auseinandergesetzt hat. Im fünften Kapitel wird näher auf die Parallelen im Zeit- und Geschichtsverständnis von Dean und Smithson eingegangen und dabei auch Kublers Einfluss berücksichtigt werden.

84 Ebd., S. 51 .

85 Gottfried Boehm, »Kunst versus Geschichte: ein unerledigtes Problem. Zur Einleitung in George Kublers >Die Form der Zeit««, in: Kubler, Die Form der Zeit, S. 7-26, S. 16.

86 Kubler, Die Form der Zeit, S. 46.

87 Ebd., S. 47. 
Der bereits erwähnte Aufsatz »Bild und Zeit« von Gottfried Boehm soll im Folgenden noch einmal kurz in seinen Grundthesen skizziert werden, weil das Verhältnis von Zeitlichkeit und Bild hier auf einer formalästhetischen Ebene konkret zur Sprache kommt. Boehm stellt die Erfahrung von Zeit im Bild in den Mittelpunkt seiner Untersuchung. Er geht vom statischen Bildwerk aus (Zeit als »die Grundkategorie der Malerei« ${ }^{88}$ ) und verfolgt das Ziel, die Prävalenz des Raumes im bildnerischen Denken zu revidieren, jedoch ohne sich auf mathematische oder naturwissenschaftliche Erkenntnisse zur Zeit zu stützen. ${ }^{89}$ Boehm unterscheidet die »Zeit des Dargestellten« von der »Zeit der Darstellung «. Erstere dominiere in der Kunsttheorie, die bisher die Zeitdarstellung im Bild an Aspekte der Bewegung einer Figur, des Bildrhythmus oder des fruchtbaren Moments im Historienbild, der narrativ für Vergangenheit und Zukunft offengehalten werde, geknüpft habe. Boehm plädiert dafür, die Zeit der Darstellung mit einzubeziehen: Sie habe ihren Ursprung in der Wahrnehmung des Bildes als »Kontrastphänomen, das sich in der Unterscheidbarkeit von Fläche und Binnenelement zeigt und bestimmt « ${ }^{90}$ Das Bild sei immer relational, und Zeitlichkeit konstituiere sich im Zusammenspiel von Binnenelement, Fläche und dem »Hin und Her des Blicks «. ${ }^{91}$

Boehm sieht in der Bewegung des Auges, welche die Wahrnehmung der Relationen zwischen der Fläche und den Bildelementen ermöglicht, den Schlüssel zum Erkennen der >ikonischen Differenz $<$ - der visuellen Logik des Bildes, die auf einer »deiktischen, das heißt nicht-sprachlichen Differenz« beruht. ${ }^{92}$ Er schreibt: »Ohne die Teilnahme des Auges hätte dieses Wechselspiel [von Teil und Ganzem, N. S.] überhaupt keine Existenz. [...] Erforderlich ist ein Hin und Her des Blickes. Räumliche Bestimmungen in der Wahrnehmung ergeben sich demnach aus einem zeitbestimmten Prozeß; der Bildraum >zeitigt< sich. «93 Da die Bewegung des Auges und somit die Wahrnehmung des Bildes sich immer in einem zeitlichen Prozess ereignen, verwendet Boehm den

\footnotetext{
88 Boehm, »Bild und Zeit«, S. 3 .

89 Vgl. ebd.

9o Ebd., S. 9.

91 Vgl. ebd., S. 12.

92 Gottfried Boehm, »Ikonische Differenz«, in: Rheinsprung 11 -Zeitschrift für Bildkritik 1, 2011, S. 170-176, S. 170. Das Konzept der ikonischen Differenz wird in Grundzügen bereits in seinem Aufsatz »Zu einer Hermeneutik des Bildes« entwickelt, wo auch die Unhintergehbarkeit der materiellen Erscheinung des Bildes betont wird: »Vom `Sein $<$ des Bildes überhaupt zu sprechen, abgesehen von seinem Erscheinen in einem Werk, ist sinnlos.« (Gottfried Boehm, »Zu einer Hermeneutik des Bildes«, in: Hans-Georg Gadamer, Gottfried Boehm [Hg.], Seminar: die Hermeneutik und die Wissenschaften, Frankfurt am Main 1978, S. 444-471, S. 451.)

Boehm, »Bild und Zeit«, S. 11 f.
} 
Begriff der Zeitigung, den er vermutlich Heidegger entlehnt. ${ }^{94}$ Auge und Bild werden als eine »Beziehungsform « ${ }^{95}$ betrachtet, in der die Wahrnehmung des Einzelelements und des Gesamtbildes sich notwendig abwechseln: »Die Zeitlichkeit des Bildes ist, paradox gesprochen, nur um den Preis der Sukzession zu haben, d. h. der Vereinzelung der Elemente, zugleich aber auch nur unter der Bedingung eines Potentials der Simultaneität. « ${ }^{96}$ Die Temporalität des Bildes hängt für Boehm also einerseits von der visuellen Organisation des Bildes ab, andererseits vom »Zeitsinn des Betrachters«. Mit seinen Worten: »Alle Bilder arbeiten [...], strukturell gesehen, mit dem Wechselspiel eines Kontrastes zwischen kontinuierenden Momenten und diskreten Elementen, sie sind eine $>$ kontinuierliche Diskontinuität « ${ }^{97}$ und diese ist als Ereignis grundsätzlich zeitlich. Dem/r Betrachter/in komme das Vermögen zu, »aus bestimmten [...] Bildstrukturen die Erfahrung von Zeit [zu] bilde[n].« Boehms Bestimmung des Verhältnisses von Zeit und Bild betont also die gegenseitige Abhängigkeit der Zeitlichkeit der Bildstruktur und der Zeiterfahrung der Betrachtenden. Dies führt er ausschließlich im Hinblick auf die Malerei aus, unter anderem mit Bezug auf Kandinsky, Mondrian und Monet. Da es sich um eine grundsätzliche strukturelle Bestimmung einer Relation von Bild, Bildelementen und Betrachterblick als zeitliche handelt, ist diese jedoch auch auf zeitlich bewegte Bilder übertragbar.

Wie der kursorische Überblick über einige Ansätze zur Betrachtung der Zeitlichkeit in der Kunst gezeigt hat, ist immer wieder versucht worden, die enge Verzahnung von Zeit und Bild zu systematisieren und zum Beispiel in Kategorien wie historische Zeit, Betrachtungszeit und eine dem Bild in Aufbau und Komposition inhärente Zeit, hier Bildzeit oder Zeitgestalt genannt, einzuteilen. Neben ihrer historischen Verankerung als materielle Artefakte verweisen Bilder auf ihre Entstehungszusammenhänge oder durch historische Darstellungen auf zeithistorische Aspekte und Ereignisse. Dass diese nicht von der erlebten Zeit der Betrachterin oder des Betrachters getrennt werden können, ist offensichtlich, denn Rezipient/innen gehen stets eine Beziehung zum Werk ein, die von ihrer persönlichen Situation ausgeht. ${ }^{98}$ Die historische

94 Martin Heidegger beschreibt, wie die Zeitlichkeit sich zeitigt, das heißt sich jeweils vollzieht - in Vergangenheit (Gewesenheit), Gegenwart und Zukunft (vgl. Martin Heidegger, Sein und Zeit, Tübingen 2006, S. 350).

96 Ebd., S. 22.

97 Boehm, »Ikonische Differenz«, S. 171.

98 Dass Rezipient/innen und Werk stets unter bestimmten Bedingungen zusammenkommen und dass die Betrachtenden immer bereits im Werk vorgesehen sind, hat Wolfgang Kemps Forschung zur Rezeptionsästhetik in der Kunstbetrachtung hervorgehoben 
Betrachtung und somit auch die Analyse und Interpretation eines historischen Werks kann nicht objektiv sein, denn sie erfolgt vom Standpunkt der zeitgenössischen und jeweils ihrer Zeit verhafteten Rezipient/innen her. Somit ist die Historizität des Werks von der Temporalität seiner Betrachtung untrennbar. Dass auch die bei Theissing so genannte Bildzeit und die Betrachtungszeit nicht scharf zu trennen sind, unterstreicht Johannes Grave. Zwar ermögliche die Vereinigung verschiedener Zeitaspekte unter dem Begriff der Bildzeit gerade wegen der entstehenden Unschärfen einen umfassenden Blick auf die Thematik der Zeitlichkeit im Bild, ${ }^{99}$ jedoch greife sie so auch die von Theissing propagierte Dreiteilung an, die »Scheidung zwischen Betrachtungszeit und Bildzeit erweist sich damit als äußerst fragil ${ }^{100}$ Legt Grave im Weiteren den Schwerpunkt auf rezeptionsästhetische Überlegungen, soll hier das Augenmerk auf die zeitlich bewegten Bilder, also auf Film, Video, Diaprojektion und digitale Bilder gelenkt werden. Zur Temporalität des Bildes, die sich wie beschrieben auch am vermeintlich statischen Bild entdecken lässt - in phänomenologischen, narrativen, semantischen, ikonografischen, formalen, gestaltästhetischen, figurativen und technischen Aspekten -, tritt hinzu, dass sich die Temporalität zeitlich bewegter Bilder technisch-medial bestimmen lässt und sich das Werk jeweils innerhalb einer bestimmten Dauer entfaltet. Mit der Bestimmung der Zeitlichkeit in bewegten Bildern haben sich naturgemäß vor allem Vertreter der Filmtheorie und -philosophie beschäftigt, deren Untersuchungen im Folgenden mit Blick auf den Realitätsbezug und -effekt, die Gegenwärtigkeit in der Erfahrung und die Dauer des Films dargestellt werden sollen.

\section{Bild und Zeit im Film}

Auf die Frage nach dem Status des Bildes im Film und nach seiner spezifischen Zeitlichkeit kann bisher keine systematische, umfassende Untersuchung eine Antwort geben. Denn beide, die Rolle des Bildes und die Rolle der Zeit im Film, gestalten sich komplex und sind miteinander verbunden - keine kann unabhängig von der anderen betrachtet werden, da ein Film immer visuell und

(vgl. Wolfgang Kemp [Hg.], Der Betrachter ist im Bild. Kunstwissenschaft und Rezeptionsästhetik, Köln 1985; ders., »Kunstwerk und Betrachter«, in: Hans Belting, Heinrich Dilly, Wolfgang Kemp, Willibald Sauerländer, Martin Warnke [Hg.], Kunstgeschichte. Eine Einführung, 7., überarbeitete und erweiterte Auflage, Berlin 20o8, S. 247-265).

»Theissings >Bildzeit< umfasst sowohl zeitliche Eigenschaften des Dargestellten als auch temporale Qualitäten der Darstellungsmittel; neben der Ikonographie und der Bildnarration berührt sie daher auch formale und bildstrukturelle Fragen.« (Grave, »Der Akt des Bildbetrachtens «, S. 53.)

Ebd., S. 54 . 
auditiv mit bewegten Bildern wahrgenommen wird und sich als zeitbasiertes Medium immer in der Zeit ablaufend ereignet. Zeit und Bild scheinen sich so also gegenseitig zu bedingen, und dieses Verhältnis soll hier mithilfe von Rückgriffen auf einzelne Autoren beleuchtet werden.

Spricht man vom Bild im Film, müssen sich Konkretisierungen anschließen: Ist mit dem Filmbild das einzelne Standbild auf dem Filmstreifen, der durch den Projektor bewegt wird, gemeint, also ein minimaler Ausschnitt aus einer Bewegung? Oder das projizierte, auf einer Leinwand sichtbar gemachte Bild? Ist als Bild nur ein einzelner Schnitt durch die kinematografische Bewegung zu bezeichnen oder die filmische Einstellung, das heißt die kleinste, mittels Schnitt und Montage erstellte Einheit eines Films? Wie unterscheidet sich schließlich ein auf dem Medium Film basierendes Bild von einer Videoprojektion oder digital erstellten Bildern? Des Weiteren muss nach dem Verhältnis des bewegten Bildes zur Zeit gefragt werden: Äußert sich diese nur in der Bewegung des Bildes (und wo ist diese Bewegung zu fassen, im Apparat oder im Bild selbst)? Ist die Zeit des Films kontinuierlich wie die Bewegung des Filmstreifens in der Kamera und im Projektor oder diskontinuierlich, da durch Schnitt und Montage des Filmmaterials die einheitliche Zeit der Realität in der filmischen Wiedergabe künstlich verändert, gestaucht oder gedehnt wird? Welche Rolle spielt die kontinuierliche Filmwahrnehmung der Betrachter/ innen, deren Imagination und Erinnerungsvermögen aus dem Bildgeschehen eine zusammenhängende Geschichte konstruieren? Und in welchem Verhältnis steht die Darstellung in einem kinematografischen Bewegungsbild zur Wirklichkeit?

Mit vielen dieser Fragen haben sich bereits frühe Filmtheoretiker beschäftigt, wobei stets vom fotografischen bewegten Bild ausgegangen wird und nicht etwa von gezeichneter Animation. André Bazin betonte in seiner »Ontologie des photographischen Bildes« die besondere Objektivität, die dem fotografischen Bild aufgrund seiner automatischen Entstehung eigen sei. Film erscheint ihm wie »die Vollendung der photographischen Objektivität in der Zeit. [...] Zum ersten Mal ist das Bild der Dinge auch das ihrer Dauer, es ist gleichsam die Mumie der Veränderung. «101 Bazin spricht in seinem kurzen Essay über das besondere Verhältnis, das das fotografische Bild mit der Wirklichkeit verbinde, und seinen daraus resultierenden Realismus im Vergleich zur Malerei. Die Kunst, also die Malerei, habe zwar die magische Funktion antiker Statuen und Mumien eingebüßt, die Lebenden vor der Zeit, das heißt dem Tod, zu schützen, »[d]och diese Entwicklung konnte das unbezwingbare

101 André Bazin, »Ontologie des photographischen Bildes« (1945), in: ders., Was ist Film?, Berlin 2004, S. 33-42, S. 39. 
Bedürfnis, die Zeit zu bannen, nur sublimieren, um es logischem Denken anzupassen «. ${ }^{102}$ Die Entwicklung der Fotografie und des Films sei die Konsequenz aus dem Streben nach Ähnlichkeit, und diese sei auch für die Ästhetik des fotografischen Bildes bestimmend: »Das ästhetische Wirkungsvermögen der Photographie liegt in der Enthüllung des Wirklichen. «103 Bazin spielt hier auf einen Topos an, den bereits Henry Fox Talbot benannt hatte und der von Autoren wie Walter Benjamin, Béla Balázs, Siegfried Kracauer, Roland Barthes und Philippe Dubois wieder aufgenommen wurde: die Möglichkeit der Fotografie und des Films, dem bloßen Auge Nicht-Sichtbares zu enthüllen, und dies beruhend auf der intrinsischen Verbindung des fotografischen Bildes zur Wirklichkeit, die auch fotografischer Index genannt wird. ${ }^{104}$ Der Automatismus der apparativen Entstehung des Bildes stelle ein privilegiertes Verhältnis zur Wirklichkeit her, welches die Realität des abgebildeten Gegenstandes nachweisen könne, »der ja tatsächlich wiedergegeben, das heißt in Raum und Zeit wieder gegenwärtig gemacht wird «. ${ }^{105}$ Bazin bezeichnet dies als »Wirklichkeitsübertragung « von der Vergangenheit in die Gegenwart. Mit Blick auf die Zeitlichkeit ist die Verwendung archäologischer Metaphern bemerkenswert: während der Film, wie bereits erwähnt, die »Mumie der Veränderung « sei, balsamiere die Fotografie die Zeit ein und entziehe sie bloß ihrem Verfall. ${ }^{106}$

Bazins Ontologie des fotografischen Bildes wird unter anderem von Siegfried Kracauer aufgegriffen, der diese als Affinität des Films zur sichtbaren Welt bezeichnet. Seine Theorie des Films vertritt eine »materiale Ästhetik « und »beruht auf der Annahme, daß der Film im wesentlichen eine Erweiterung der Fotografie ist und daher mit diesem Medium eine ausgesprochene Affinität zur sichtbaren Welt um uns herum gemeinsam hat «.107 Kracauer beschäftigt sich mit den Inhalten des Films und leitet aus dessen medialen Eigenschaften

\footnotetext{
102 Ebd., S. 34.

103 Ebd., S. 39 .

104 Der Index ist bekanntlich keine genuin fotografische Kategorie, sondern ein Begriff aus der Semiotik nach Charles Sanders Peirce, dem die Fotografie als ein Beispiel unter vielen für die Beschreibung seiner verschiedenen Zeichentypen dient. Indexikalität bezeichnet demnach das kausale, physikalische Beziehungsverhältnis einer Sache zu ihrem Ursprung (im Gegensatz zum Ikon und zum Symbol). Dieses Verhältnis kann eine Ähnlichkeit der Fotografie zum dargestellten Objekt beinhalten, muss dies aber nicht. (Zur Karriere der Indexikalität im fotografischen Diskurs und ihrer Kritik siehe zusammenfassend Peter Geimer, Theorien der Fotografie zur Einführung, Hamburg 2009, Kapitel 1: »Bilder durch Berührung. Fotografie als Abdruck, Spur und Index«, S. 13-69.)

105 Bazin, »Ontologie des photographischen Bildes«, S. 37.

106 Ebd., S. 39 .

107 Siegfried Kracauer, Theorie des Films. Die Errettung der äußeren Wirklichkeit, hg. v. Karsten Witte, Frankfurt am Main 1985 (engl. Orig. 196o), S. 11.
} 
die Privilegierung der Dinge ab, die er allein darzustellen vermag: »[D]as Kino [scheint] von dem Wunsch beseelt, vorübergleitendes materielles Leben festzuhalten, Leben in seiner vergänglichsten Form. «108 Dementsprechend kritisiert Kracauer den Kunstanspruch des Films, denn er sei der Oberfläche der Dinge verpflichtet, man verkenne seine Bestimmung, würde man ihn nicht die sichtbaren Phänomene um ihrer selbst willen aufzeichnen lassen.109 Bazin und Kracauer werden der >realistischen< Strömung innerhalb der Filmtheorie zugeordnet, die das Gegenstück zur `formalistischen < bildet. Während die realistische Strömung einen dem Film immanenten »Durchblick auf eine (nicht-mediale) Wirklichkeit« betont, sehen die Formalisten, zu denen neben Sergej Eisenstein als Hauptvertreter auch Rudolf Arnheim, die russischen Formalisten und die amerikanischen Neoformalisten David Bordwell, Kristin Thompson und Noël Carroll gezählt werden können, den Film primär als Konstruktion und Repräsentation an. ${ }^{110}$ So können hier auch zwei Zugänge zur Zeit im Film unterschieden werden: einerseits die Repräsentation von Zeit und zeitlichen Abläufen beispielsweise in der Bewegung, wie sie sich in der gefilmten Realität ereignet haben, andererseits die Gestaltung der Zeiterfahrung durch den/die Betrachter/in mittels filmischer Mittel wie Kamerabewegung, Schnitt und Montage. Beide Aspekte lassen sich nicht trennen und treten in unterschiedlicher Ausprägung in jedem filmischen Werk auf.

108 Ebd.

109 Vgl. ebd., S. 13. Auf dieses in der Kamerarealität begründete Apriori, das zugleich ein Werturteil ist und ein bestimmtes Verständnis von Realismus begründet, wird zum Beispiel im Hinblick auf die Arbeiten von Tacita Dean zurückzukommen sein. Ihre Arbeitsweise ist von einer ähnlichen Affinität zur ungestellten Realität gekennzeichnet.

110 Vgl. Thomas Elsaesser, Malte Hagener, Filmtheorie zur Einführung, Hamburg 2007, S. 10. Inwiefern die Unterscheidung in zwei Haupttendenzen, die Kracauer bereits bei Lumière und Mélies als dem »strikten Realisten« und dem Filmemacher, »der seiner künstlerischen Fantasie freien Lauf ließ « beschrieb (Kracauer, Theorie des Films, S. 57), für eine Klassifikation innerhalb der Filmtheorie zielführend ist, ist fraglich, zumal die implizite Wertung zu einseitigen Betrachtungsweisen und zur Verkennung vielschichtiger Werke führen kann, wie Franz-Josef Albersmeier anhand des Realismusstreits mit Blick auf das Gesamtwerk Sergej Eisensteins erläutert hat. Dessen psychosoziologischer Ansatz wurde zugunsten seiner Montagetheorie in den Hintergrund gedrängt (vgl. Franz-Josef Albersmeier, »Einleitung. Filmtheorien in historischem Wandel«, in: ders. [Hg.], Texte zur Theorie des Films, 5., durchgesehene und erweiterte Auflage, Stuttgart 2003, S. 3-29, S. 13 f.). Ebenso lässt sich eine geografische Unterteilung in deutsch-, französisch- und englischsprachige Autoren wählen sowie die Einteilung in Schulen. Albersmeier stellt für die neuere Filmtheorie drei schwerpunktmäßige Forschungskomplexe fest: Psychologisch und/oder psychoanalytisch orientierte Filmtheorien, in die auch feministische Ansätze einfließen, philosophische Fragestellungen im Gewand der Filmtheorie und schließlich inter- und multimedial angelegte Filmtheorien (vgl. ebd., S. 18 f.). 
Die einflussreichsten Bücher der Filmphilosophie zur Erforschung der Zeit im Bild sind zweifellos die beiden sogenannten Kino-Büchervon Gilles Deleuze. In ihnen verknüpft Deleuze eine $» \operatorname{Tax}[\mathrm{o}]$ nomie, ein[en] Klassifizierungsversuch der Bilder und Zeichen «111 mit filmhistorischen Betrachtungen. Anhand der Unterscheidung zwischen Vor- und Nachkriegskino, also dem Kino seit der Stummfilmzeit bis etwa zum Ausbruch des Zweiten Weltkriegs und dem Kino des Neorealismus und der Nouvelle Vague bis hin zum Experimentalfilm und der Videokunst, denen er einen jeweils radikal unterschiedlichen Umgang mit Bewegung und Zeit im Bild bescheinigt, beschreibt Deleuze zwei verschiedene Konzeptionen des bewegten Bildes und, wie Raymond Bellour feststellt, orientiert diese auch an unterschiedlichen philosophischen Weltentwürfen, der klassischen und der modernen Philosophie. ${ }^{112}$ Die Kino-Bücher sind keine filmhistorischen Werke, wenngleich sie einer groben historischen Einteilung folgen, sondern es werden in ihnen unterschiedliche Bildtypen auf der Grundlage ihrer Beziehung zur Zeit charakterisiert. Im ersten Band, dem Bewegungs-Bild. Kino I, der 1983 auf Französisch und 1989 auf Deutsch erschien, steht zunächst ein grundsätzliches Nachdenken über Bewegung im Mittelpunkt. Deleuze bezieht sich auf Henri Bergson, dem er die Entdeckung des Bewegungs- wie auch des Zeit-Bildes zuschreibt, ${ }^{113}$ wenngleich er sich in seinen Thesen zur Bewegung gegen dessen Definition der kinematografischen Bewegung als »kinematographische Illusion «114 wendet. Er bezieht sich dabei auf die Bände Materie und Gedächtnis (1896) und Schöpferische Entwicklung (1907), in denen Bergson feststellt, dass Bewegung im Gegensatz zum Raum, der sich aus einzelnen Punkten rekonstruieren lässt, nicht teilbar ist, sondern sich vielmehr immer in einer konkreten zeitlichen Dauer ereignet. ${ }^{115}$ Bergson beschreibt das neue, kinematografische Medium als Vermittler falscher Bewegungen, da hier aus der Kopplung von stillstehenden Bildern und Bewegung nur die Illusion von Bewegung erzeugt werde. ${ }^{116}$ Deleuze entgegnet dem, dass jedoch der Film, auch wenn er mit Phasenbildern arbeitet,

111 Gilles Deleuze, Das Bewegungs-Bild. Kino I, Frankfurt am Main 1989, S. 11.

112 Vgl. Raymond Bellour, »Denken, Erzählen. Das Kino von Gilles Deleuze«, in: Oliver Fahle (Hg.), Der Film bei Deleuze / Le cinéma selon Deleuze, Weimar/Paris 1997, S. 41-6o, S. 52.

113 Deleuze, Das Bewegungs-Bild, S. 11.

114 Ebd., S. 14.

115 Vgl. ebd., S. 13.

116 Bergson vergleicht die Schwierigkeit, eine Veränderung, ein Werden zu denken (und den Vorgang des Denkens selbst) mit dem kinematografischen Verfahren und dessen unnatürlicher, weil auf einem Apparat beruhender Rekonstruktion von Bewegung: »Die Bewegung entschlüpft in das Intervall, weil jeder Versuch, Veränderung aus Zuständen zu rekonstruieren, die sinnlose Voraussetzung einschließt, Bewegung bestehe aus Unbewegtheiten.« (Henri Bergson, Schöpferische Entwicklung, Zürich 1967, S. 305.) 
uns kein »Photogramm, sondern ein Durchschnittsbild [gibt], dem dann nicht etwa noch Bewegung hinzugefügt oder hinzugezählt würde - Bewegung ist im Gegenteil im Durchschnittsbild unmittelbar gegeben « ${ }^{117}$ Man dürfe von der Künstlichkeit der Mittel nicht auf die Künstlichkeit des Ergebnisses schließen, denn »der Film gibt uns kein Bild, das er dann zusätzlich in Bewegung brächte - er gibt uns unmittelbar ein Bewegungsbild «.118 Bewegungs-Bilder sind also »Bilder, die sich nur in und durch Bewegung konstituieren und damit ihre Existenz als Bild nur in Verbindung mit einer Bewegung finden, die ihr Auftauchen und Verlöschen reguliert «. ${ }^{119}$ Sie sind immer ephemer und limitiert, wie Mirjam Schaub feststellt, und zwischen materiellem Filmbild und Vorstellungsbild anzusiedeln - »am ehesten jene projizierten Bilder, die wir als Nachbilder der Leinwand auf unserer Retina sehen. Unsere Augen sind das ausführende und sich selbst sakkadenhaft bewegende Organ, das die Bewegung der Bilder nicht bloß aktualisiert, sondern genuin konstituiert.« ${ }^{120}$ Bewegungsbilder werden im Weiteren in verschiedene Bildtypen unterteilt (Wahrnehmungsbild, Aktionsbild und Affektbild), ${ }^{121}$ die sich in Bezug darauf unterscheiden, »wie unvermittelt und wie sichtbar sie vom Reiz zur Reaktion gelangen «, ${ }^{122}$ also wie eng die Verbindung zwischen den eine Handlung auslösenden Aktionen/ Bildern/Reizen und der darauf folgenden Reaktion ist. Der Abstand, der bei einem Aktionsbild am kürzesten und deutlichsten ist und in dessen »sensomotorisches Bild « ${ }^{123}$ der Zuschauer / die Zuschauerin per Identifikation mit der Figur hineingezogen wird, wird beim Wahrnehmungsbild bereits fühlbar ausgedehnt und nimmt die falschen Anschlüsse des Zeit-Bilds vorweg. Jenen Wechsel in der Konzeption des Kino-Bildes stellt Deleuze im zweiten KinoBuch, das 1985 (auf Deutsch 1991) erschien, einleitend anhand verschiedener Szenen neorealistischer Bilder wie folgt dar: »Wir haben es nunmehr mit einem Kino des Sehenden und nicht mehr mit einem Kino der Aktion zu tun.« Und weiter: »Man muss von einer neuen Art von Zeichen sprechen, nämlich von Opto- und Sonozeichen «, ${ }^{124}$ die auf ganz unterschiedliche Arten von Bildern und filmischen Situationen verweisen. In diesen tritt zum Realen des Bildes seine mentale und imaginäre Ebene, sie werden ununterscheidbar,

\footnotetext{
117 Deleuze, Das Bewegungs-Bild, S. 14.

118 Ebd., S. 15 .

119 Mirjam Schaub, Gilles Deleuze im Kino. Das Sichtbare und das Sagbare, München 2003, S. 92 .

120 Ebd., S. 93 .

121 Vgl. Deleuze, Das Bewegungs-Bild, S. 94-96.

122 Schaub, Gilles Deleuze im Kino, S. 95.

123 Gilles Deleuze, Das Zeit-Bild. Kino II, Frankfurt am Main 1997, S. 13.

124 Ebd., S. 17.
} 
was beispielsweise an Alain Resnais' L'Année dernière à Marienbad (1961) zu beobachten ist. ${ }^{125}$ Die Zeit spielt für das Zeit-Bild eine stärkere Rolle als für das Bewegungs-Bild, in dem sie mittels der Bewegung stets an die spezifische Situation und Aktion gebunden war. Bellour fasst die Unterscheidung sehr treffend wie folgt zusammen:

Ersteres [das Bewegungs-Bild, N. S.] beschreibt das Kino als eine einheitliche Welt, die aus >rationalen Schnitten $<$ zwischen den Einstellungen hergestellt wird, die nach den jeweiligen Montagearten ein indirektes Bild der Zeit ergeben, das sich auf sensomotorische Schemata stützt und dank einer Kontinuität zwischen Aktion und Reaktion eine organische Beziehung zwischen den einzelnen Teilen und dem >Ganzen< stiftet - einem Ganzen, das zugleich das Ganze des Bildes wie auch das mögliche Ganze der Welt ist. Das moderne Kino hingegen [das ZeitBild, N. S.] basiert auf Brüchen, auf >irrationalen Schnitten<, die einen neuen, nicht faßbaren Zwischenraum zwischen den Einstellungen deutlich machen. Die Handlungen sind nicht mehr von einem Reiz-Reaktions-System bestimmt, sondern unterliegen einem allgemeinen Phänomen der Unbeweglichkeit und des Sehens, das einen direkten Zugang zur Zeit erlaubt, mithin ein direktes Bild der Zeit ermöglicht. ${ }^{126}$

Bei Yasujiro Ozu beobachtet Deleuze die Zunahme toter Zeiten und leerer Räume; als er die Dauer einer gefilmten Vase beschreibt und den Wandel von Tag zur Nacht im Dämmerlicht - Einstellungen, die einem Stillleben nahekommen -, charakterisiert er sie als »die Zeit selbst«, direkte Zeit-Bilder, die sich genau deshalb von einer Fotografie unterscheiden, weil sich die Veränderung in ihrer »unwandelbaren Form « ereignet: »In dem Augenblick, in dem das kinematographische Bild dem Photo am nächsten kommt, unterscheidet es sich zugleich am radikalsten von ihm. «127

Mirjam Schaub konzentriert sich in ihrer detaillierten Analyse von Deleuzes Zeit-Bild auf den Umgang mit Begrifflichkeiten, die Zeit und Aspekte von Zeitlichkeit benennen. Dass Deleuze die Beschreibung der Zeit im konkreten Film erfolgreich mit philosophischen und bildtheoretischen Überlegungen verknüpft hat, macht seine Gedanken so fruchtbar für die vorliegende Untersuchung, weil sie sich als auf kinematografische Installationen übertragbar erweisen. Als Grundideen des Zeit-Bildes nennt Schaub dreierlei: 1. Das sich in der Krise befindende Nachkriegskino erfährt seine Rettung durch die Zeitphilosophie, das heißt, auch der Nachkriegsfilm fungiert, wie Schaub im Anschluss an Bellour argumentiert, als eine Art »verkleideter und verkappter

125 Vgl. ebd., S. 19.

126 Bellour, »Denken, Erzählen«, S. 43.

127 Deleuze, Das Zeit-Bild, S. 31. 
Philosophiegeschichte ${ }^{128}$ 2. Die Gegenwärtigkeit und Aktualität des Filmbildes wird - ausgehend vom Gegenmodell des rein Virtuellen - einer Kritik unterzogen. 3. Filmbilder thematisieren Zeit anders als die gesprochene oder geschriebene Sprache. ${ }^{129}$ Interessant für die Beschreibung und Analyse der Zeitlichkeit des filmischen Bildes ist vor allem die zweite Feststellung, nämlich Deleuzes Wendung gegen die Gegenwärtigkeit des Filmbildes, welche sich an verschiedenen Stellen im Zeit-Bild belegen lässt und von ihm auch in Interviews bestätigt wurde. So wenig wie die Gegenwärtigkeit eines Bildes an seine Projektion zu koppeln sei, sei die Zuschreibung von Vergangenheit und Zukunft im Bild an narrative Konventionen zu binden: »Die Gegenwart des Bildes ist ihm nicht gegeben, sondern sie muß, wie alle anderen Zeitformen auch, behauptet und über Indizes repräsentiert werden. ${ }^{130}$ Zeit im Bild sei demnach immer inszeniert, immer produziert, es gebe keine Zeitform, die im filmischen Bild privilegiert sei. Die »Gegenwart als einzige reale Zeitform im Medium des Bildes « ${ }^{131}$ will Deleuze aufheben, da er von einem relationalen Zeitbegriff ausgeht: »[I]l me semble évident que l'image n'est pas au présent. Ce qui est au présent, c'est ce que l'image >représente`, mais pas l'image ellemême. L'image même, c'est un ensemble de rapports de temps dont le présent ne fait que découler, soit comme commun multiple, soit comme plus petit diviseur.«132

Für Deleuze steht die Virtualität des Bildes im Vordergrund, das heißt ein Zustand, der von der realen Gegenwart und demnach auch von der Wahrnehmung durch Betrachter/innen unabhängig ist. Das Bild sei »eine zeitliche Relationsordnung, voller divergenter Momente «, 133 beschreibt Schaub, und es habe daher keine »genuin zeitliche Verfassung «.134 Dennoch oder gerade deshalb ist es dem kinematografischen Bild nach Deleuze möglich, äußerst komplexe zeitliche Konstellationen darzustellen wie beispielsweise in

128 Schaub, Gilles Deleuze im Kino, S. 121. Es handele sich diesmal jedoch um einen doppelten Zeitbegriff, »Zeit als continens et contentum, als Bedingtes und Bedingendes, als vorübergehende (gegenwärtige) und sich bewahrende (rein virtuelle). In dem Maße, in dem sich Zeit vom Raum als theoretischem Stichwortgeber emanzipiert, läßt sich die moderne, nachkantische Sicht auf Zeitlichkeit in der Entwicklung des kinematographischen Bildes mitverfolgen, so Deleuzes Idee.« (Ebd., S. 122.)

129 Ebd., S. 121-126.

130 Ebd., S. $124 \mathrm{f}$.

131 Ebd., S. 125.

132 Deleuze in: Pascal Bonitzer, Jean Narboni, » Le cerveau, c'est l'écran<. Entretien avec Gilles Deleuze«, in: Cahiers du Cinéma 380, 1986, S. 25-36, S. 32, zit. n. Schaub, Gilles Deleuze im Kino, S. 124.

133 Ebd., S. 125.

134 Ebd., S. 126. 
seiner Konzeption des Kristallbildes, das zugleich aktuell und virtuell ist, in einer internen Spaltung beziehungsweise Doppelung (beispielsweise mittels Spiegelungen, die das Beobachtete verunklären, oder in der Rolle des Schauspielers, der zugleich Person und Rolle ist). Dass das Kristallbild auch als Zustand zweier unterschiedlicher Zeitlichkeiten im Bild beschrieben werden kann, als simultane Evokation von Vergangenheit und Gegenwart, wird später anhand des Werkes Box (ahareturnabout) (1977) von James Coleman erläutert.

Deleuzes Ablehnung einer gegenwärtigen Dimension des Kinobildes verwundert nicht, zieht man in Betracht, dass er die phänomenologische Ebene der Filmerfahrung außen vor lässt, da sie in seiner Gleichsetzung von Kino und Denken keine Rolle spielt. Dabei ist die Rolle der Betrachter/innen, ihre physische Präsenz, intellektuelle, kognitive und psychische Beteiligung am Bildgeschehen sowie soziale Prägung und individuelle Erinnerung für den Film spätestens seit dem Aufkommen der ideologiekritischen und psychoanalytischen Filmtheorie der 1970er und 1980er Jahre intensiv diskutiert worden. Christian Metz, dessen Theorie des Films als Sprache von Deleuze als zu stark dem Strukturalismus verhaftet zurückgewiesen wird, ${ }^{135}$ hat in einem Essay zum Realitätseindruck des Films die Bewegung als dasjenige Element des Films hervorgehoben, das ihm Leben verleiht und bei den Zuschauer/innen den Eindruck von Realität, die Möglichkeit des Wiedererkennens des Realen, hervorruft. Die Bewegung werde dabei stets als gegenwärtig wahrgenommen, nicht etwa als "Spur einer vergangenen Bewegung«, wie man in Bezug auf die Fotografie vermuten würde:

135 Deleuze orientiert sich, wie bereits auf der ersten Seite des Bewegungs-Bildes formuliert, in seiner Taxonomie der filmischen Zeichen an der Semiotik von Charles Sanders Pierce. D. N. Rodowick erklärt Deleuzes Kritik an der Semiologie, vor allem derjenigen Christian Metz', damit, dass die Sprache als grammatikalische Struktur zu starr und unbeweglich sei und die Materialität und Zeitlichkeit des Bildes unbeobachtet lasse: »The critique of Metz focuses on two basic themes - immanence and temporality - that are central to Deleuze's critique of structuralism in general. Structuralism ignores the materiality of different materials of expression by reducing them to a universal >structure $<$, the system of linguistic signs. Moreover, this universal logic is understood fundamentally as static and unchanging. For Deleuze, Peirce's semiotics restores a sense of the temporality of signs in relation to thought, as well as their immanence with respect to the materials of expression that supports them.«(D. N. Rodowick, Gilles Deleuze's Time Machine, Durham/ London 1997, S. 40.) Die Narration ergebe sich beispielsweise im Kino nicht aus der zugrunde liegenden sprachlichen Struktur, sondern aus den Bildern selbst: »Die Erzählhandlung ist niemals eine sichtbare Gegebenheit der Bilder oder die Wirkung einer ihnen zugrunde liegenden Struktur; vielmehr ist sie eine Konsequenz der selbst sichtbaren und von sich aus wahrnehmbaren Bilder, so wie sie sich zunächst als solche bestimmen.« (Deleuze, Das Zeit-Bild, S. 43, vgl. Rodowick, Gilles Deleuze's Time Machine, S. 41.) 
[D]er Zuschauer nimmt die Bewegung immer als gegenwärtig auf (selbst wenn sie eine vergangene Bewegung >reproduziert<), so daß die >zeitliche Ausgeglichenheit<, von der Roland Barthes spricht - dieser Eindruck des >früher<, der den Anblick einer Photographie irrealisiert -, aufhört, bei dem Anblick einer Bewegung eine Rolle zu spielen. ${ }^{136}$

Es ist für jedes filmische Werk von großer Bedeutung, ob das Dargestellte von dem Betrachter oder der Betrachterin als gegenwärtig oder als vergangen, als real oder als irreal wahrgenommen wird, da entsprechend die Partizipation am Gesehenen beziehungsweise die Distanznahme zum Bildgeschehen unbewusst erfolgen. Für die hier besprochenen Werke wird der Aspekt der Gegenwärtigkeit an den Stellen interessant, wo die gezielte Einbeziehung der Zuschauer/innen erfolgt oder andersherum die Teilhabe am Bildgeschehen aktiv vermieden wird, um eine kritische Distanz zu erwirken und dadurch eine Reflexion nicht nur der erzählten filmischen Inhalte, sondern auch der formalen ästhetischen Komponenten des Werks.

Die technisch-medialen Bedingungen des Films berücksichtigt Gilles Deleuze in seiner Kinophilosophie nicht, da er das Bild von seiner virtuellen Dimension her denkt. Andere Autoren hingegen knüpfen die Frage nach der Besonderheit des filmischen Bildes vor allem an seine technische Bewegung, wie zum Beispiel Noël Carroll: Der Eindruck von Bewegung muss für ihn technisch erzeugt werden können, was Animations- und Fotofilme mit einschließt, jedoch den Unterschied des bewegten Bildes zur Malerei oder anderen Künsten hervorhebt. ${ }^{137}$ Ebenso bezieht sich Joachim Paech in seinen zahlreichen Aufsätzen zum Bewegungsbild auf den mechanischen Ursprung des Films, also die Tatsache, dass die Einzelbilder des Films mittels Hemmung in einer bestimmten Frequenz durch die Lichtquelle des Projektors gezogen werden und auf der Leinwand zu einem einzelnen bewegten Bild verschmelzen. Dort sehen wir Bewegung als »Differenz-Figur ihrer Darstellung «, 138 die Figurierung nämlich der Differenz zwischen den einzelnen Bildern erscheint uns als einheitliche, realistische Bewegung. Diese wird nicht etwa als Täuschung oder Überlagerung von einzelnen Bildern im Auge erzeugt, sondern figuriert bereits in der Projektion auf der Leinwand. Ob und wie Bewegung gesehen wird, hängt nach Paech von der Beziehung der figurativen Darstellung der einzelnen

136 Christian Metz, »Zum Realitätseindruck im Kino« (1965), in: ders., Semiologie des Films, München 1972, S. 20-34, S. 27.

137 Vgl. Noël Carroll, Theorizing the Moving Image, Cambridge 1996, S. 63-66.

138 Joachim Paech, »Bewegung als Figur und Figuration (in Photographie und Film)«, in: Gottfried Boehm, Maja Naef, Achatz von Müller (Hg.), Figur und Figuration. Studien zu Wahrnehmung und Wissen, Paderborn 2007, S. 275-291, S. 277. 
Bilder untereinander ab: Gibt es figurative Veränderungen von Bild zu Bild, werden diese Veränderungen als Bewegung gesehen; sind die Veränderungen zu groß, nehmen wir sie als Sprünge und Unterbrechungen der dargestellten Kontinuität der Bewegung, als sogenannte Jump-Cuts wahr, sind sie zu klein, erscheint der Film als freeze frame, als stillgestelltes Bild im Film. ${ }^{139}$ Paech geht in seinen Überlegungen zum kinematografischen Bewegungsbild häufig von der Frage nach ihrem Gegenteil aus: der Stillstellung von Bewegung im Film, dem arrêt sur l'image, den Raymond Bellour 1987 als einen Zustand von Zeitlichkeit des Bildes benennt, welchen Gilles Deleuze in seiner Taxonomie der Bilder unberücksichtigt lässt. ${ }^{140}$ Mit Blick auf die Zeitlichkeit ist dieses Phänomen bemerkenswert, verknüpft es doch die grundsätzlich unterschiedlichen Verhältnisse zur Zeit in Fotografie und Film: Das Verhältnis zur Zeit ist in der Fotografie stärker »augenblickskritisch«, im Film eher »verlaufs- oder dauerkritisch «, abhängig von der jeweiligen temporalen Definition des Bildes im Bezug zum Moment der Entstehung beziehungsweise dem Verlauf der zeitlichen Erstreckung, die Lorenz Engell in seinem Aufsatz zum arrêt sur l'image unterscheidet. ${ }^{141}$ Engell betont die Gegenwärtigkeit des Films:

Der Film thematisiert nicht die zeitliche Beziehung zwischen dem Abgebildeten und dem Bild selbst, sondern diejenige der Bilder untereinander. [...] Das Filmbild gibt nicht, wie die Fotografie, den gewesenen Augenblick wieder, sondern die je bewegliche, in jedem Jetzt neu aktualisierte Differenz zwischen Vergangenheit und Zukunft. [...] So, wie die Fotografie grundsätzlich im Modus der Vergangenheit operiert, so operiert der Film stets in demjenigen der andauernden Gegenwart und der gegenwärtigen Dauer. ${ }^{142}$

Dies sei vollkommen unabhängig von der Zeitsemantik des Films, das heißt der Darstellung von Vergangenheit. So argumentiert auch Stanley Cavell, der den Zeitcharakter des Films unabhängig von der Tatsache, ob es sich um Fiktion oder Geschichte handelt, daran knüpft, dass die Zuschauer/innen bei

139 Joachim Paech, »Medienwissenschaft«, in: Klaus Sachs-Hombach, Bildwissenschaft. Disziplinen, Themen, Methoden, Frankfurt am Main 2005, S. 78-96, S. 88.

140 Raymond Bellour, »L'interruption, l'instant« (1987), in: ders. (Hg.), L'Entre-Images, S. 109-133, S. 110. Bellour beschreibt verschiedene Beispiele, in denen sich die Fotografie in den Film >einschleicht< und auf die noch zurückzukommen sein wird.

141 Lorenz Engell, »AAre you in pictures? R Ruhende Bilder am Ende bewegter Bilder, besonders in Ethan und Joel Coens BARTON FINK«, in: Stefanie Diekmann, Winfried Gerling (Hg.), Freeze Frames. Zum Verhältnis von Fotografie und Film, Bielefeld 2010, S. 172-191, S. 173.

142 Ebd., S. 18o f. Die Dauer ist also der vorfilmischen wie auch der filmischen Realität eigen, denn wie Bazin bereits beschrieb: »Zum ersten Mal ist das Bild der Dinge auch das ihrer Dauer« (Bazin, »Ontologie des photographischen Bildes«, S. 39). 
etwas anwesend seien, das selbst abwesend, weil vergangen sei. ${ }^{143}$ Der Film ist paradoxerweise präsent, er zeigt seine Welt dem/der Betrachter/in stets gegenwärtig, ist aber auch immer schon vergangen. Diese doppelte Zeitstruktur, die jedes Filmbild kennzeichnet, ist deshalb spezifisch filmisch, weil sie eben auch eine spezifische Dauer hat. Diese unterscheidet sie von der Fotografie, die als Augenblicksbild stets in die Vergangenheit verweist und für deren Zeitcharakter die Indexikalität eine weit größere Rolle spielt. Der Film hat eine spezifische Gegenwärtigkeit, die in der Wahrnehmung der kinematografischen Bewegung durch den Betrachter oder die Betrachterin gründet, in der Tatsache, dass Bewegung immer als gegenwärtig wahrgenommen wird; zugleich zeigt er immer Vergangenes und hat eine intrinsische Verbindung zur Geschichte beziehungsweise zur Geschichtlichkeit des Dargestellten und des Mediums selbst. Diese Verschränkung der Zeiten wird in den Werken zeitgenössischer Kunst immer wieder mit künstlerischen Mitteln explizit gemacht. Es ist zu fragen, inwiefern es auch ein zeithistorisches Phänomen ist, das von einem neuen Geschichtsbewusstsein der Künstler/innen ausgeht, welches einerseits auf eine Rückbesinnung auf die Materialität der Kunst angesichts der Virtualisierung der Historie und andererseits auf den Einbruch historischer Umbrüche in die Realität der Menschen zurückzuführen ist.

\section{Fazit}

Die zitierten kunsthistorischen Positionen konzentrieren sich auf die historische Zeit als Entstehungszeit des Bildes, auf die Bildzeit als imaginäre Zeit des Bildes (die Zeitstruktur), die Rezeptionszeit der Betrachter/innen, die gegenseitige Abhängigkeit von Bildstruktur und Zeiterfahrung oder, wie bei Kubler, die Wahrnehmung der Zeitlichkeit der Dinge im Kontext ihrer diskontinuierlichen Geschichtsbezüge. Zeitlichkeit zeigt sich hier vor allem an dem Zusammenwirken der Darstellung von Bewegung oder der bildlichen Erzählung mit der Zeit der Betrachtung. Im zeitlich bewegten Bild des Films tritt die Verlaufszeit des Films hinzu, welche die Zeit der Rezeption mit der Dauer der Projektion, der eventuellen Zeit der Erzählung und der materialästhetischen, technischen Zeit konfrontiert. Die Wahrnehmung der Bewegung im Film hebt dessen Gegenwärtigkeit hervor; zugleich muss das besondere Verhältnis des Bildes zur Wirklichkeit über den fotografischen Index berücksichtigt werden. Das Zeit-Bild nach Deleuze stellt einen eigenen Entwurf dar, in welchem dem aktuellen Bild stets auch eine Virtualität innewohnt, so dass das Verhältnis der Zeiten im Bild zueinander als relational beschrieben wird.

143 Stanley Cavell, »Welt durch die Kamera gesehen«, in: Dieter Henrich, Wolfgang Iser (Hg.), Theorien der Kunst, Frankfurt am Main 1982, S. 447-49o, S. 478. 
Relationalität kennzeichnet auch nach Boehm das statische Bild, jedoch hinsichtlich des Verhältnisses von Zeit und Raum, die mittels der Blickbewegung verbunden werden: »[D]er Bildraum >zeitigt« sich «, ${ }^{144}$ das heißt, erst durch die »zeitliche Erfassung der Elemente ${ }^{145}$ können auch die räumlichen Gegebenheiten bestimmt und Zeit und Raum des Bildes als Ganzes wahrgenommen werden.

Die Gegenüberstellung von Zeit- und Raumkünsten nach Lessing wird spätestens mit der Erfindung des Films obsolet. Dessen spezifische Möglichkeiten lassen sich, wie Panofsky es am eingängigsten formuliert hat, als »Dynamisierung des Raumes und entsprechend als Verräumlichung der Zeit« ${ }^{146}$ definieren. Aus Panofskys Essay über den Kunststatus des Films ist diese Gegenüberstellung das meistzitierte Diktum; »evident bis zur Selbstverständlichkeit « ${ }^{147}$ ist vor allem der erste Teil, denn durch die bewegliche Kamera, Einstellungen und Montage ist das Auge in Identifikation mit der Linse stets in Bewegung. Die Feststellung, dass die Zeit sich verräumliche, hat laut Thomas Meder hingegen theoretische Brisanz, denn die Materialisierung der Zeit sei nicht sichtbar, sondern nur kognitiv verständlich und nur symbolisch in den Bildern enthalten: »Beide Zeitarten, die Darstellung in der realen Zeit und die dargestellte Zeit, haben nur bedingt miteinander zu tun: Jede Symbolisierung von Zeit verändert die reale und normale Zeiterfahrung, beide werden im räumlichen Modus aber in eigenartiger W[ei]se erfahrbar.«148

Um die Besonderheit des multimedialen, bewegten Bildes und sein Verhältnis zur Zeit anschaulich zu machen, möchte ich kurz den Blick auf eine Ausstellungssituation lenken, die 1999 in Berlin zu sehen war: Im Foyer der Neuen Nationalgalerie, dem gläsernen Mies-van-der-Rohe-Gebäude am Potsdamer Platz, befanden sich zwei großformatige, durchscheinende Leinwände. Bei Tageslicht war nur schemenhaft zu erkennen, dass darauf etwas gezeigt wurde, nachts jedoch konnte man von außen deutlich sehen, dass hier zwei filmische Arbeiten projiziert wurden (Abb. 3). Die eine zeigte - auf den ersten Blick - ein beliebiges Standbild des Films The Searchers von John Ford, eines Technicolor-Westerns aus dem Jahr 1956. Das andere zeigte eine verwackelte Videoaufnahme von Andy Warhols Film Empire (1966). Beides sind Werke von

\footnotetext{
144 Boehm, »Bild und Zeit«, S. 12.

145 Ebd.

146 Erwin Panofsky, »Stil und Medium im Film« (engl. 1947), in: ders., Die ideologischen Vorläufer des Rolls-Royce-Kühlers \& Stil und Medium im Film, Frankfurt am Main, Campus 1993, S. 17-51, S. 22.

147 Ebd.

148 Thomas Meder, Produzent ist der Zuschauer. Prolegomena zu einer historischen Bildwissenschaft des Films, Berlin 2006, S. 166.
} 


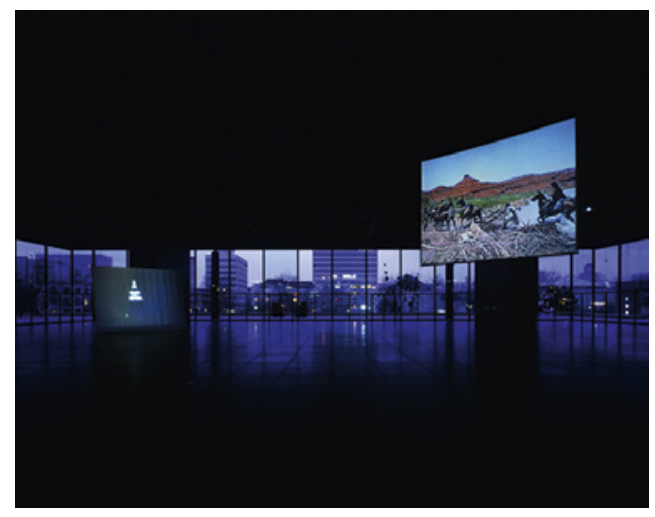

Abb. 3

Douglas Gordon, 5 Year Drive-By v. Bootleg (Empire), Ausstellungsansicht Neue Nationalgalerie, Berlin, 1999

Douglas Gordon, in denen er sich wie bei 24 Hour Psycho vorhandenes Filmmaterial aneignet und die neben ihren vordergründigen Bezügen zum Hollywoodkino und zum Filmgebrauch der Avantgarde einige Aspekte des Bildes in der kinematografischen Projektion veranschaulichen. Die Arbeit 5 Year Drive-By (1995), in der The Searchers als Videoversion adaptiert wird, zeigt tatsächlich kein Standbild, sondern den Film so stark verlangsamt, dass er sich, zeigte man die Installation komplett, auf die fünf Jahre erstrecken würde, die die in der Erzählung kondensierte Handlung umfasst. ${ }^{149}$ Das bedeutet, das einzelne Videobild ist für jeweils 14 Minuten zu sehen, der Bildwechsel meist kaum wahrnehmbar. 5 Year Drive-By treibt so das projizierte Videobild in einen Zwischenraum von (adaptiertem) Film, Video, Fotografie und Malerei, berücksichtigt man die auffällige Farbigkeit des Films und die Reminiszenzen John Fords an Traditionen der Landschaftsmalerei. ${ }^{150}$ Es ist kein freeze frame, der durch die Wiederholung eines Einzelbildes entsteht, da Gordon mit Video arbeitet und so ein Medium verwendet, dessen Bildgenerierung auf dem stets erneuerten elektronischen Signal beruht. Und im Gegensatz zu Warhols Empire entsteht Stillstand hier auch nicht deshalb, weil etwas Unbewegtes wie das Empire State Building gefilmt wurde. Das Anhalten auf dem Videobild lässt jede kleine Veränderung plötzlich bedeutend werden, und der Bildwechsel wird zum dramatischen Moment, in welchem den Betrachter/innen die Bewegung des Films in ihrer Abwesenheit bewusst wird. Gordon unterläuft durch seine Dehnung der Filmzeit die Elemente der filmischen Wahrnehmung, die oben angesprochen wurden: die Erfahrung des Films als gegenwärtig

\footnotetext{
149 In der Handlung begibt sich John Wayne in seiner Figur des Ethan Edwards auf eine fünf Jahre dauernde Suche nach seiner Nichte, die von Indianern entführt wurde.

$15^{\circ}$ Meder, Produzent ist der Zuschauer, S. 358.
} 
durch die Bewegung sowie die Beziehung der Einzelbilder und Einstellungen zueinander durch Schnitt und Montage. Durch die veränderte räumliche Situation im Ausstellungskontext rückt das Werk in die Nähe des statischen Bildes; es ermöglicht eine freie Bewegung des Auges, ein >Hin und Her des Blicks im Verlauf der Betrachtung, aus dem sich die zeitliche Erfahrung des einzelnen Frames ergibt. Die einzelnen projizierten Bilder bieten sich dem Auge als Zwitterwesen zwischen Bewegt- und Standbild an: Bilder, die im Hinblick auf Bildaufbau, Farbe, Linienführung und Motivik analysiert und interpretiert werden können und so jeweils für sich wirken, eine eigene Zeitgestalt entwickeln; die jedoch auch untrennbar vom Kontext der Filmerzählung sind. ${ }^{151}$ In der Adaption des Films von 1956 durch Gordon im Jahr 1998 mittels einer genauen mathematischen Kalkulation, wie lange jeder Frame gezeigt werden muss, um den Film auf die fünf Jahre auszudehnen - eine Methode, die an den strukturellen Film der 197oer Jahre erinnert ${ }^{152}$-, schafft Gordon einen historischen Rahmen für seine Arbeit. Der Film hat einerseits einen konkreten räumlichen und zeitlichen Bezug zu einer Landschaft, die durch Fords Filme untrennbar mit seinem Namen und somit auch mit einer spezifischen Tradition des amerikanischen Westerns verknüpft ist; andererseits hebt die Überhöhung dieser Bilder durch Gordon mittels der reinen Zeitspanne, in der sie jeweils gezeigt werden, jeden historischen und filmimmanenten Verweisungszusammenhang auf. ${ }^{153}$ Durch die Monumentalisierung des Einzelbildes geht seine Aussagekraft innerhalb der Filmerzählung verloren, zugleich stellt sich jedoch die Frage nach seiner jeweils eigenen diegetischen Dimension. Dies entspricht jedoch nicht der von Frey behaupteten transzendenten Überzeitlichkeit künstlerischer Werke. Vielmehr wird das Werk durch die Wahrnehmung des Betrachters / der Betrachterin an die Realität der Betrachtung rückgebunden. Das schmerzliche Aushalten der immensen, unmöglich im Ganzen erlebbaren Zeitspanne und die Kontemplation, die irgendwann in gespanntes Warten oder Langeweile umschlägt, verankern das Werk in der Gegenwart.

151 Siehe ausführlich zur Motivik von The Searchers Elsaesser, Hagener, Filmtheorie zur Einführung, Kapitel 2 (»Tür und Leinwand«), sowie Meder, Produzent ist der Zuschauer, S. 372 f.

152 Vgl. Philip Monk, Double-Cross. The Hollywood-Films of Douglas Gordon, Toronto 2003, S. 84 f. Auf den strukturellen Film und seine Parallelen zu Werken der zeitgenössischen kinematografischen Installation wird weiter unten erneut eingegangen.

153 Monk nennt sie »zeitlich erhaben« im Gegensatz zum »räumlich Erhabenen«, das Ford mit den Landschaftsaufnahmen erzeuge: »In Gordon's project, Ford's spatial sublime is supplanted by a temporal sublime.« (Ebd., S. 91.) 
Setzt man die bisher betrachteten Werkbeispiele - Line of Faith von James Coleman sowie die beiden Spielfilmadaptionen von Douglas Gordon - in Bezug zu den skizzierten methodischen Ansätzen aus der Kunst-, Film- und Medienwissenschaft, wird deutlich, dass die kinematografischen Installationen in ihrer Verschränkung von verschiedenen Zeitebenen die Methoden und Begrifflichkeiten herausfordern. Mit zeitlichen Manipulationen wie hier der extremen Verlangsamung, aber auch mit dispositiven Anordnungen, der räumlichen Nutzung von Split Screens oder der Irritation der Betrachter/ innen durch Jump Cuts und falsche Anschlüsse in der Montage werden die Erzählung und Einbeziehung der Betrachtenden über die Identifikation mit Bewegung, Handlung und Charakteren dekonstruiert. Die starke Bezugnahme auf historische Ereignisse und künstlerische Traditionen sowie der kreative Gebrauch technischer Medien, der die semantischen Konnotationen von Film, Fotografie, Video und Malerei hinterfragt und neu bestimmt, führten in den letzten zwei Jahrzehnten zu neuen Ansätzen, multimediale Werke zu beschreiben. Begrifflichkeiten wie >Zeitstrukturく oder >Zeitgestalt Bildes, die das Verhältnis der Zeit der Darstellung zur Zeit des Dargestellten beschreiben, sind hier weiterhin hilfreich. Sie erlauben es, die Rolle der Betracher/innen, die in den kinematografischen Installationen als verbindendes Element von Raum-, Zeit- und Bilderfahrung eine wichtige Position einnehmen, zu berücksichtigen. Das Verhältnis der Zeit der Darstellung zur Zeit des Dargestellten gewinnt noch an Komplexität, da zusätzlich die zeitliche Erstreckung des bewegten Bildes und somit eine technisch-mediale Zeit berücksichtigt werden muss. Die Zeitlichkeit der Medien und der Bilder in Bezug zu ihrer Geschichtlichkeit wird in der jüngeren Forschung anhand von Konzepten wie Inframedialität, ${ }^{154}$ entre-image und obsolescence (Veralterung) ${ }^{155}$ gefasst; die Projektion ist als Medium, das den Ausstellungsraum >verzeitlicht< und das Bild >verräumlicht<, in den Blick gerückt. ${ }^{156}$ Gordon

154 Ilka Becker (Hg.), Just not in time. Inframedialität und non-lineare Zeitlichkeiten in Kunst, Film, Literatur und Philosophie, München 2011. Der Neologismus Inframedialität soll nicht das Verhältnis zwischen den Medien beschreiben, sondern im Sinne eines topologischen Modells »Brüche, Schwellen, Verschiebungen und Transformationen von Vielheit im Inneren medialer Konfigurationen und vor allem auch innerhalb von Einzelmedien« thematisieren (vgl. Ilka Becker, Michael Cuntz, Michael Wetzel, »Einleitung«, in: ebd., S. $7-27$, S. 11).

155 Malcolm Turvey, Annette Michelson, Ken Jacobs, »Round Table. Obsolescence and American Avant-Garde Film «, in: October 100/1, 2002, S. 115-132.

156 Vgl. Stan Douglas, Christopher Eamon (Hg.), Art of Projection, Ostfildern 2009; Lilian Haberer, Annette Urban (Hg.), Bildprojektionen. Filmisch-fotografische Dispositive in Kunst und Architektur, Bielefeld, 2016, darin auch mein Aufsatz »>To ferry images of presence and absence $\lessdot$. Projektion, Medium und Bild bei James Coleman und Douglas Gordon«, S. 117-130. 
reflektiert die fiktionalen, narrativen und illusionistischen Mittel des Spielfilms durch seine Verwendung der Medien Video und Projektion, die die traditionellen Konventionen der Filmwahrnehmung im Kinoraum und der Präsentation von Kunstwerken im Ausstellungsraum einander gegenüberstellen. Die historische Dimension in Form der Bezugnahme auf die Geschichte des Westerns, der Landschaftsmalerei und die Methoden des Experimentalfilms ist für die Arbeit unmittelbar von Bedeutung und mit der Zeitlichkeit in einer produktiven Dichotomie verbunden. Line of Faith zeigt eindrücklich, dass mittels der Reinszenierung und Reproduktion die historische Fiktionalisierung der Currier-and-Ives-Lithografie den Charakter eines Dokuments annimmt; Colemans Werk rückt dabei die Frage nach dem Evidenzcharakter der Fotografie und des Videos, nach ihrer realitätsbezeugenden Funktion in den Blick. Colemans und Gordons Arbeiten zeigen, dass hier die Geschichtlichkeit der Medien Fotografie, Film und Video in ihrer Bedeutung für die Kunst der Moderne reflektiert wird und die Rezeption über die Zeitlichkeit des Bildes erfolgt. Sie können ebenso wie die in den folgenden Kapiteln vorgestellten Arbeiten als Beispiele dafür dienen, wie in der Gegenwartskunst die formalästhetische Arbeit mittels der Zeitlichkeit des Bildes mit ihrer Geschichtlichkeit zusammenfällt. 